\title{
Paths and tableaux descriptions of Jacobi-Trudi determinant associated with quantum affine algebra of type $D_{n}$
}

\author{
Wakako Nakai • Tomoki Nakanishi
}

Received: 7 March 2006 / Accepted: 8 January 2007 /

Published online: 7 April 2007

(C) Springer Science+Business Media, LLC 2007

\begin{abstract}
We study the Jacobi-Trudi-type determinant which is conjectured to be the $q$-character of a certain, in many cases irreducible, finite-dimensional representation of the quantum affine algebra of type $D_{n}$. Unlike the $A_{n}$ and $B_{n}$ cases, a simple application of the Gessel-Viennot path method does not yield an expression of the determinant by a positive sum over a set of tuples of paths. However, applying an additional involution and a deformation of paths, we obtain an expression by a positive sum over a set of tuples of paths, which is naturally translated into the one over a set of tableaux on a skew diagram.
\end{abstract}

Keywords Quantum group $\cdot q$-character $\cdot$ Lattice path $\cdot$ Young tableau

\section{Introduction}

Let $\mathfrak{g}$ be the simple Lie algebra over $\mathbb{C}$, and let $\hat{\mathfrak{g}}$ be the corresponding untwisted affine Lie algebra. Let $U_{q}(\hat{\mathfrak{g}})$ be the quantum affine algebra, namely, the quantized universal enveloping algebra of $\hat{\mathfrak{g}}[4,11]$. In order to investigate the finite-dimensional representations of $U_{q}(\hat{\mathfrak{g}})[3,5]$, an injective ring homomorphism

$$
\chi_{q}: \operatorname{Rep} U_{q}(\hat{\mathfrak{g}}) \rightarrow \mathbb{Z}\left[Y_{i, a}^{ \pm 1}\right]_{i=1, \ldots, n ; a \in \mathbb{C}^{\times}},
$$

called the $q$-character of $U_{q}(\hat{\mathfrak{g}})$, was introduced and studied in [6,7], where $\operatorname{Rep} U_{q}(\hat{\mathfrak{g}})$ is the Grothendieck ring of the category of the finite-dimensional representations of $U_{q}(\hat{\mathfrak{g}})$. The $q$-character contains the essential data of each representation $V$. So far, however, the explicit description of $\chi_{q}(V)$ is available only for a

W. Nakai $(\varangle) \cdot$ T. Nakanishi

Graduate School of Mathematics, Nagoya University, Nagoya 464-8602, Japan

e-mail: m99013c@math.nagoya-u.ac.jp

T. Nakanishi

e-mail: nakanisi@math.nagoya-u.ac.jp 
limited type of representations (e.g., the fundamental representations) [2, 6], and the description for general $V$ is an open problem. See [10, 19] for related results.

In our previous work [17], for $\mathfrak{g}=A_{n}, B_{n}, C_{n}$, and $D_{n}$, we conjecture that the $q$-characters of a certain family of, in many cases irreducible, finite-dimensional representations are given by the determinant form $\chi_{\lambda / \mu, a}$, where $\lambda / \mu$ is a skew diagram and $a$ is a complex parameter. We call $\chi_{\lambda / \mu, a}$ the Jacobi-Trudi determinant. For $A_{n}$ and $B_{n}$, this is a reinterpretation of the conjecture for the spectra of the transfer matrices of the vertex models associated with the corresponding representations $[1,14]$. See also [15] for related results for $C_{n}$ and $D_{n}$. Let us briefly summarize the result of [17]. Following the standard Gessel-Viennot method [9], we represent the JacobiTrudi determinant by paths, and apply an involution for intersecting paths. For $A_{n}$ and $B_{n}$, this immediately reproduces the known tableaux descriptions of $\chi_{\lambda / \mu, a}$ by $[1,14]$. Here, by tableaux description we mean an expression of $\chi_{\lambda / \mu, a}$ by a positive sum over a certain set of tableaux on $\lambda / \mu$. For $A_{n}$, the relevant tableaux are nothing but the semistandard tableaux as the usual character for $\mathfrak{g}=A_{n}$. For $B_{n}$, the tableaux are given by the 'horizontal' and 'vertical' rules similar to the ones for the semistandard tableaux. In contrast, we find that the tableaux description of $\chi_{\lambda / \mu, a}$ for $C_{n}$ is not as simple as the former cases. The main difference is that a simple application of the Gessel-Viennot method does not yield an expression of $\chi_{\lambda / \mu, a}$ by a positive sum. Nevertheless, in some special cases (i.e., a skew diagram $\lambda / \mu$ of at most two columns or of at most three rows), one can further work out the cancellation of the remaining negative contribution, and obtain a tableaux description of $\chi_{\lambda / \mu, a}$. Besides the horizontal and vertical rules, we have an additional rule, which we call the extra rule, due to the above process.

In this paper, we consider the same problem for $\chi_{\lambda / \mu, a}$ for $D_{n}$, where the situation is quite parallel to $C_{n}$. By extending the idea of [17], we now successfully obtain a tableaux description of $\chi_{\lambda / \mu, a}$ for a general skew diagram $\lambda / \mu$. The resulting tableaux description shows nice compatibility with the proposed algorithm to generate the $q$-character by [6], and it is expected to be useful to study the $q$-characters further. We also hope that our tableaux will be useful to parameterize the much-awaited crystal basis for the Kirillov-Reshetikhin representations $[12,20]$, where $\lambda / \mu$ is a rectangular shape. To support it, for a two-row rectangular diagram $\lambda / \mu$, our tableaux agree with the ones for the proposed crystal graph by [21]. Meanwhile, our tableau rule is rather different from the one for the non-quantum case [8] due to the different nature of the determinant and the generating function. The method herein is also applicable to a general skew diagram $\lambda / \mu$ for $C_{n}$, and it will be reported in a separate publication [18].

Now let us explain the organization and the main idea of the paper.

In Sect. 2, following [17], we define the Jacobi-Trudi determinant $\chi_{\lambda / \mu, a}$ for $D_{n}$. The procedure to derive the tableaux description of $\chi_{\lambda / \mu, a}$ consists of three steps.

In Sect. 3 we do the first step. Here we apply the standard method by [9] for the determinant $\chi_{\lambda / \mu, a}$. Namely, first, we introduce lattice paths, and express $\chi_{\lambda / \mu, a}$ as a sum over a set of $l$-tuples of paths $p=\left(p_{i}\right)$ with fixed end points. Secondly, we define the weight-preserving, sign-reversing involution $\iota_{1}$ (the first involution) so that for an intersecting tuple of paths $p$ the contributions from $p$ and $\iota_{1}(p)$ cancel each other in the sum. Unlike $A_{n}$ and $B_{n}$, however, this involution cannot be defined on the 
entire set of the intersecting tuples of paths, and the resulting expression for $\chi_{\lambda / \mu, a}$ (the first sum, Proposition 3.2) still includes negative terms.

In Sect. 4 we do the second step. Extending the idea of [17] for $C_{n}$, we define another weight-preserving, sign-reversing involution $\iota_{2}$ (the second involution). Then, the resulting expression (the second sum, Theorem 4.13) no longer includes negative terms. However, the contribution from the tuples of paths with 'transposed' pairs still remains, and one cannot naturally translate such a tuple of paths into a tableau on the skew diagram $\lambda / \mu$.

In Sects. 5 and 6, we do the last step. In Sect. 5, we claim the existence of a weight-preserving deformation $\phi$ of the paths (the folding map), where $\phi$ 'resolves' transposed pairs by folding. The resulting expression (the third sum, Theorem 5.2) is now naturally translated into the tableaux description whose tableaux are determined by the horizontal, vertical, and extra rules (Theorems 5.6 and 5.8). The explicit list of the extra rule has wide variety, and examples are given for $\lambda / \mu$ with at most two columns or at most three rows. The construction of the folding map $\phi$ is the most technical part of the work. We provide the details in Sect. 6.

We remark that while the explicit list of the extra rule for tableaux looks rather complicated and disordered, it is a simple and easily recognizable graphical rule in the path language. Therefore, the paths description (especially, the third sum) may be as important as the tableaux description for applications.

\section{The Jacobi-Trudi determinant of type $D_{n}$}

In this section, we define the Jacobi-Trudi determinant $\chi_{\lambda / \mu, a}$, following [17]. See [17] for more information.

A partition is a sequence of weakly decreasing non-negative integers $\lambda=$ $\left(\lambda_{1}, \lambda_{2}, \ldots\right)$ with finitely many non-zero terms $\lambda_{1} \geq \lambda_{2} \geq \cdots \geq \lambda_{l}>0$. The length $l(\lambda)$ of $\lambda$ is the number of the non-zero integers. The conjugate of $\lambda$ is denoted by $\lambda^{\prime}=\left(\lambda_{1}^{\prime}, \lambda_{2}^{\prime}, \ldots\right)$. As usual, we identify a partition $\lambda$ with a Young diagram $\lambda=\left\{(i, j) \in \mathbb{N}^{2} \mid 1 \leq j \leq \lambda_{i}\right\}$, and also identify a pair of partitions such that $\lambda_{i} \geq \mu_{i}$ for any $i$, with a skew diagram $\lambda / \mu=\left\{(i, j) \in \mathbb{N}^{2} \mid \mu_{i}+1 \leq j \leq \lambda_{i}\right\}$.

Let

$$
I=\{1,2, \ldots, n, \bar{n}, \ldots, \overline{2}, \overline{1}\} .
$$

Let $\mathcal{Z}$ be the commutative ring over $\mathbb{Z}$ generated by $z_{i, a}$ 's, $i \in I, a \in \mathbb{C}$, with the following generating relations:

$$
z_{i, a} z_{\bar{i}, a-2 n+2 i}=z_{i-1, a} z_{\overline{i-1}, a-2 n+2 i} \quad(i=1, \ldots, n), \quad z_{0, a}=z_{\overline{0}, a}=1 .
$$

Let $\mathbb{Z}[[X]]$ be the formal power series ring over $\mathbb{Z}$ with variable $X$. Let $\mathcal{A}$ be the non-commutative ring generated by $\mathcal{Z}$ and $\mathbb{Z}[[X]]$ with relations

$$
X z_{i, a}=z_{i, a-2} X, \quad i \in I, a \in \mathbb{C} .
$$

For any $a \in \mathbb{C}$, we define $E_{a}(z, X), H_{a}(z, X) \in \mathcal{A}$ as 


$$
\begin{gathered}
E_{a}(z, X):=\left\{\prod_{1 \leq k \leq n}\left(1+z_{k, a} X\right)\right\}\left(1-z_{\bar{n}, a} X z_{n, a} X\right)^{-1}\left\{\prod_{1 \leq k \leq n}^{\leftarrow}\left(1+z_{\bar{k}, a} X\right)\right\}, \\
H_{a}(z, X):=\left\{\overrightarrow{\prod_{1 \leq k \leq n}}\left(1-z_{\bar{k}, a} X\right)^{-1}\right\}\left(1-z_{\bar{n}, a} X z_{n, a} X\right)\left\{\prod_{1 \leq k \leq n}^{\leftarrow}\left(1-z_{k, a} X\right)^{-1}\right\},
\end{gathered}
$$

where $\vec{\prod}_{1 \leq k \leq n} A_{k}=A_{1} \ldots A_{n}$ and $\prod_{1 \leq k \leq n} A_{k}=A_{n} \ldots A_{1}$. Then we have

$$
H_{a}(z, X) E_{a}(z,-X)=E_{a}(z,-X) H_{a}(z, X)=1 .
$$

For any $i \in \mathbb{Z}$ and $a \in \mathbb{C}$, we define $e_{i, a}, h_{i, a} \in \mathcal{Z}$ as

$$
E_{a}(z, X)=\sum_{i=0}^{\infty} e_{i, a} X^{i}, \quad H_{a}(z, X)=\sum_{i=0}^{\infty} h_{i, a} X^{i},
$$

with $e_{i, a}=h_{i, a}=0$ for $i<0$.

Due to relation $(2.5)$, we have [16, (2.9)]

$$
\operatorname{det}\left(h_{\lambda_{i}-\mu_{j}-i+j, a+2\left(\lambda_{i}-i\right)}\right)_{1 \leq i, j \leq l}=\operatorname{det}\left(e_{\lambda_{i}^{\prime}-\mu_{j}^{\prime}-i+j, a-2\left(\mu_{j}^{\prime}-j+1\right)}\right)_{1 \leq i, j \leq l^{\prime}}
$$

for any pair of partitions $(\lambda, \mu)$, where $l$ and $l^{\prime}$ are any non-negative integers such that $l \geq l(\lambda), l(\mu)$ and $l^{\prime} \geq l\left(\lambda^{\prime}\right), l\left(\mu^{\prime}\right)$. For any skew diagram $\lambda / \mu$, let $\chi_{\lambda / \mu, a}$ denote the determinant on the left- or right-hand side of (2.6). We call it the Jacobi-Trudi determinant associated with the quantum affine algebra $U_{q}(\hat{\mathfrak{g}})$ of type $D_{n}$.

Let $d(\lambda / \mu):=\max \left\{\lambda_{i}^{\prime}-\mu_{i}^{\prime}\right\}$ be the depth of $\lambda / \mu$. We conjecture that, if $d(\lambda / \mu) \leq n$, the determinant $\chi_{\lambda / \mu, a}$ is the $q$-character for a certain finite-dimensional representations $V$ of quantum affine algebras. We further expect that $\chi_{\lambda / \mu, a}$ is the $q$-character for an irreducible $V$, if $d(\lambda / \mu) \leq n-1$ and $\lambda / \mu$ is connected [17].

Remark 2.1 The above conjecture and the ones for types $B_{n}$ and $C_{n}$ in [17] tell that the irreducible character of $U_{q}(\hat{\mathfrak{g}})$, corresponding to a connected skew diagram, is always expressed by the same determinant (2.6) regardless of the type of the algebra. This is a remarkable contrast to the non-quantum case [13]. For example, the tensor product of two first fundamental modules of $\mathfrak{g}$ has two irreducible submodules for type $A_{n}$ and three ones for type $B_{n}, C_{n}$, or $D_{n}$. On the other hand, under the appropriate choice of the values for the spectral parameters, the tensor product of two first fundamental representations of $U_{q}(\hat{\mathfrak{g}})$ has exactly two irreducible subquotients, one of which corresponds to two-by-one rectangular diagram and the other of which corresponds to one-by-two rectangular diagram, regardless of the type of the algebra. In fact, this is the simplest example of the conjecture.

\section{Gessel-Viennot paths and the first involution}

Following [17], let us apply the method by [9] to the determinant $\chi_{\lambda / \mu, a}$ in (2.6) and the generating function $E_{a}(z, X)$ in (2.3). 


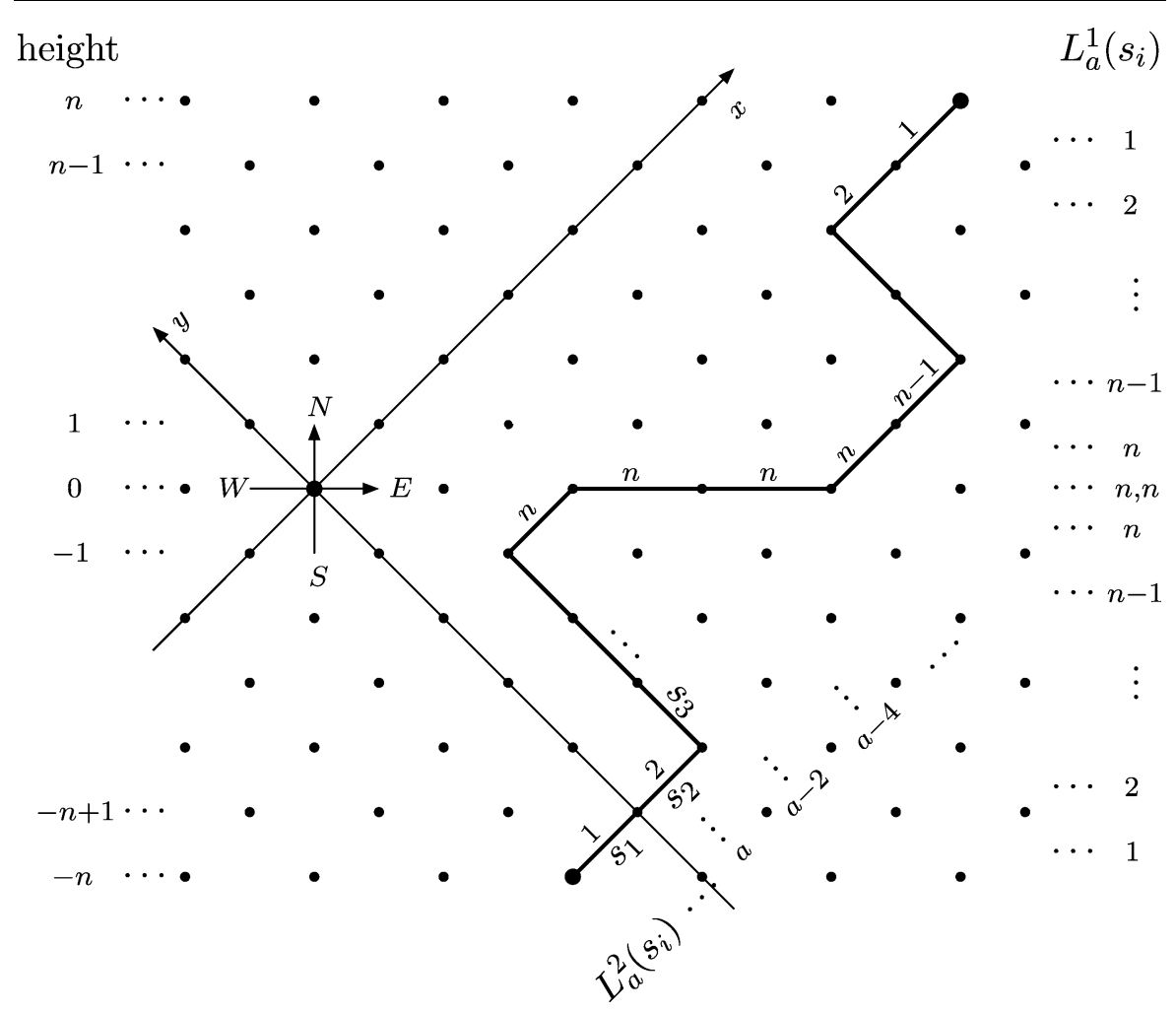

Fig. 1 An example of a path of type $D_{n}$ and its $e$-labeling

Consider the lattice $\mathbb{Z}^{2}$ and rotate it by $45^{\circ}$ as in Fig. 1. An E-step $s$ is a step between two points in the lattice of length $\sqrt{2}$ in east direction. Similarly, an $N E$ step (resp. an NW-step) is a step between two points in the lattice of unit length in northeast direction (resp. northwest direction). For any point $(x, y) \in \mathbb{R}^{2}$, we define the height as $h t(x, y):=x+y$, and the horizontal position as $h p(x, y):=\frac{1}{2}(x-y)$. Due to (2.3), we define a path $p$ (of type $D_{n}$ ) as a sequence of consecutive steps $\left(s_{1}, s_{2}, \ldots\right)$ which satisfies the following conditions:

(1) It starts from a point $u$ at height $-n$ and ends at a point $v$ at height $n$.

(2) Each step $s_{i}$ is an NE-, NW-, or E-step.

(3) The E-steps occur only at height 0 , and the number of E-steps is even.

We also write $p$ as $u \stackrel{p}{\rightarrow} v$. See Fig. 1 for an example.

Let $\mathcal{P}$ be the set of all paths of type $D_{n}$. For any $p \in \mathcal{P}$, set

$$
\begin{aligned}
E(p) & :=\{s \in p \mid s \text { is an NE- or E-step }\} \\
E_{0}(p) & :=\{s \in p \mid s \text { is an E-step }\} \subset E(p) .
\end{aligned}
$$

If $E_{0}(p)=\left\{s_{j}, s_{j+1}, \ldots, s_{j+2 k-1}\right\}$, then let

$$
E_{0}^{1}(p):=\left\{s_{j+1}, s_{j+3}, \ldots, s_{j+2 k-1}\right\} \subset E_{0}(p) .
$$


Fix $a \in \mathbb{C}$. The $e$-labeling (of type $D_{n}$ ) associated with $a$ for a path $p \in \mathcal{P}$ is the pair of maps $L_{a}=\left(L_{a}^{1}, L_{a}^{2}\right)$ on $E(p)$ defined as follows: Suppose that a step $s \in E(p)$ starts at a point $w=(x, y)$, and let $m:=\operatorname{ht}(w)$. Then, we set

$$
\begin{aligned}
L_{a}^{1}(s) & = \begin{cases}n+1+m, & \text { if } m<0, \\
n, & \text { if } m=0 \text { and } s \in E_{0}^{1}(p), \\
\overline{n-m}, & \text { otherwise }\end{cases} \\
L_{a}^{2}(s) & =a-2 x .
\end{aligned}
$$

See Fig. 1.

Now we define the weight of $p \in \mathcal{P}$ as

$$
z_{a}^{p}:=\prod_{s \in E(p)} z_{L_{a}^{1}(s), L_{a}^{2}(s)} \in \mathcal{Z} .
$$

By the definition of $E_{a}(z, X)$ in (2.3), for any $k \in \mathbb{Z}$, we have

$$
e_{r, a-2 k}(z)=\sum_{p} z_{a}^{p}
$$

where the sum runs over all $p \in \mathcal{P}$ such that $(k,-n-k) \stackrel{p}{\rightarrow}(k+r, n-k-r)$.

For any $l$-tuples of distinct points $u=\left(u_{1}, \ldots, u_{l}\right)$ of height $-n$ and $v=$ $\left(v_{1}, \ldots, v_{l}\right)$ of height $n$, and any permutation $\sigma \in \mathfrak{S}_{l}$, let

$$
\mathfrak{P}(\sigma ; u, v):=\left\{p=\left(p_{1}, \ldots, p_{l}\right) \mid p_{i} \in \mathcal{P}, u_{i} \stackrel{p_{i}}{\rightarrow} v_{\sigma(i)} \text { for } i=1, \ldots, l\right\},
$$

and set

$$
\mathfrak{P}(u, v):=\bigsqcup_{\sigma \in \mathfrak{S}_{l}} \mathfrak{P}(\sigma ; u, v) .
$$

We define the weight $z_{a}^{p}$ and the sign $(-1)^{p}$ of $p \in \mathfrak{P}(u, v)$ as

$$
z_{a}^{p}:=\prod_{i=1}^{l} z_{a}^{p_{i}}, \quad(-1)^{p}:=\operatorname{sgn} \sigma \quad \text { if } p \in \mathfrak{P}(\sigma ; u, v) .
$$

For any skew diagram $\lambda / \mu$, set $l=\lambda_{1}$, and

$$
\begin{array}{ll}
u_{\mu}:=\left(u_{1}, \ldots, u_{l}\right), & u_{i}:=\left(\mu_{i}^{\prime}+1-i,-n-\mu_{i}^{\prime}-1+i\right), \\
v_{\lambda}:=\left(v_{1}, \ldots, v_{l}\right), & v_{i}:=\left(\lambda_{i}^{\prime}+1-i, n-\lambda_{i}^{\prime}-1+i\right) .
\end{array}
$$

Then, due to (3.3), the determinant (2.6) can be written as

$$
\chi_{\lambda / \mu, a}=\sum_{p \in \mathfrak{P}\left(u_{\mu}, v_{\lambda}\right)}(-1)^{p} z_{a}^{p}
$$


In the $A_{n}$ case, one can define a natural weight-preserving, sign-reversing involution on the set of all the tuples $p$ which have some intersecting pair $\left(p_{i}, p_{j}\right)$. However, this does not hold for $D_{n}$ because of Condition (3) of the definition of a path of type $D_{n}$. Therefore, as in the cases of types $B_{n}$ and $C_{n}$ [17], we introduce the following notion:

Definition 3.1 We say that an intersecting pair $\left(p_{i}, p_{j}\right)$ of paths is specially intersecting if it satisfies the following conditions:

(1) The intersection of $p_{i}$ and $p_{j}$ occurs only at height 0 .

(2) $p_{i}(0)-p_{j}(0)$ is odd, where $p_{i}(0)$ is the horizontal position of the leftmost point on $p_{i}$ at height 0 .

Otherwise, we say that an intersecting pair $\left(p_{i}, p_{j}\right)$ is ordinarily intersecting.

As in the cases of types $B_{n}$ and $C_{n}$ [17], we can define a weight-preserving, signreversing involution $\iota_{1}$ on the set of all the tuples $p \in \mathfrak{P}\left(u_{\mu}, v_{\lambda}\right)$ which have some ordinarily intersecting pair $\left(p_{i}, p_{j}\right)$. Therefore, we have

Proposition 3.2 For any skew diagram $\lambda / \mu$,

$$
\chi_{\lambda / \mu, a}=\sum_{p \in P_{1}(\lambda / \mu)}(-1)^{p} z_{a}^{p},
$$

where $P_{1}(\lambda / \mu)$ is the set of all $p \in \mathfrak{P}\left(u_{\mu}, v_{\lambda}\right)$ which do not have any ordinarily intersecting pair $\left(p_{i}, p_{j}\right)$ of paths.

For $B_{n}$, the sum (3.6) is a positive sum because no $p \in P_{1}(\lambda / \mu)$ has a 'transposed' pair $\left(p_{i}, p_{j}\right)$. But, this is not so for $C_{n}$ and $D_{n}$.

\section{The second involution}

In this section, we define another weight-preserving involution, the second involution. This is defined by using the paths deformations called expansion and folding. As a result, the second involution cancels all the negative contributions in (3.6), and we obtain an expression by a positive sum, see (4.7).

\subsection{Expansion and folding}

Let

$$
\begin{aligned}
& S_{+}:=\left\{(x, y) \in \mathbb{R}^{2} \mid 0 \leq \operatorname{ht}(x, y) \leq n\right\}, \\
& S_{-}:=\left\{(x, y) \in \mathbb{R}^{2} \mid-n \leq \operatorname{ht}(x, y) \leq 0\right\} .
\end{aligned}
$$

For any $w=(x, y) \in S_{+}$, define $w^{*} \in S_{-}$by

$$
w^{*}:=(-y+1,-x-1) .
$$


Then we have $\operatorname{ht}\left(w^{*}\right)=-\operatorname{ht}(w), \operatorname{hp}\left(w^{*}\right)=\mathrm{hp}(w)+1$. Conversely, we define $\left(w^{*}\right)^{*}=w$, and we call the correspondence

$$
S_{+} \leftrightarrow S_{-}, \quad w \leftrightarrow w^{*}
$$

the dual map.

Definition 4.1 A lower path $\alpha$ (of type $D_{n}$ ) is a sequence of consecutive steps in $S_{-}$which starts at a point of height $-n$ and ends at a point of height 0 , and each step is an NE- or NW-step. Similarly, an upper path $\beta$ (of type $D_{n}$ ) is a sequence of consecutive steps in $S_{+}$which starts at a point of height 0 and ends at a point of height $n$, and each step is an NE- or NW-step.

For any lower path $\alpha$ and an upper path $\beta$, let $\alpha(r)$ and $\beta(r)$ be the horizontal positions of $\alpha$ and $\beta$ at height $r$, respectively. We define an upper path $\alpha^{*}$ and a lower path $\beta^{*}$ by

$$
\alpha^{*}(r)=\alpha(-r)-1, \quad \beta^{*}(-r)=\beta(r)+1, \quad(0 \leq r \leq n)
$$

and call them the duals of $\alpha, \beta$.

Let

$$
(\alpha ; \beta):=\left(\alpha_{1}, \ldots, \alpha_{l} ; \beta_{1}, \ldots, \beta_{l}\right)
$$

be a pair of an $l$-tuple $\alpha$ of lower paths and an $l$-tuple $\beta$ of upper paths. We say that $(\alpha ; \beta)$ is nonintersecting if $\left(\alpha_{i}, \alpha_{j}\right)$ is not intersecting, and so is $\left(\beta_{i}, \beta_{j}\right)$ for any $i, j$.

From now on, let $\lambda / \mu$ be a skew diagram, and we set $l=\lambda_{1}$. Let

$$
\mathcal{H}(\lambda / \mu):=\left\{\begin{array}{l|l}
(\alpha ; \beta)=\left(\alpha_{1}, \ldots, \alpha_{l} ; \beta_{1}, \ldots, \beta_{l}\right) & \begin{array}{c}
(\alpha ; \beta) \text { is nonintersecting } \\
\alpha_{i}(-n)=\frac{n}{2}+\mu_{i}^{\prime}+1-i \\
\beta_{i}(n)=-\frac{n}{2}+\lambda_{i}^{\prime}+1-i
\end{array}
\end{array}\right\}
$$

For any skew diagram $\lambda / \mu$, we call the following condition the positivity condition:

$$
\lambda_{i+1}^{\prime}-\mu_{i}^{\prime} \leq n, \quad i=1, \ldots, l-1 .
$$

We call this the 'positivity condition', because (4.2) guarantees that $\chi_{\lambda / \mu, a}$ is a positive sum (see Theorem 4.13). By the definition, we have

Lemma 4.2 Let $\lambda / \mu$ be a skew diagram satisfying the positivity condition (4.2), and let $(\alpha ; \beta) \in \mathcal{H}(\lambda / \mu)$. Then,

$$
\beta_{i+1}(n) \leq \alpha_{i}^{*}(n), \quad \beta_{i+1}^{*}(-n) \leq \alpha_{i}(-n) .
$$

A unit $U \subset S_{ \pm}$is either a unit square with its vertices on the lattice, or half of a unit square with its vertices on the lattice and the diagonal line on the boundary of $S_{ \pm}$. See Fig. 2 for examples. The height ht $(U)$ of $U$ is given by the height of the left vertex of $U$. 
Fig. 2 Examples of units

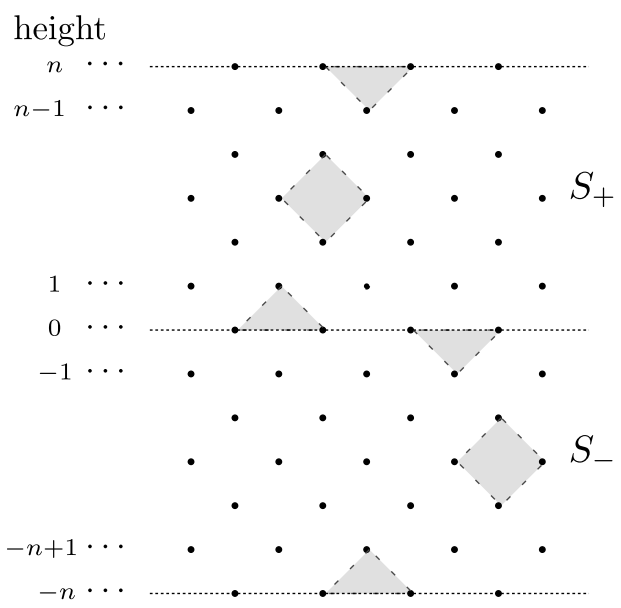

Definition 4.3 Let $(\alpha ; \beta) \in \mathcal{H}(\lambda / \mu)$. For any unit $U \subset S_{ \pm}$, let $\pm r=\operatorname{ht}(U)$ and let $a$ and $a^{\prime}=a+1$ be the horizontal positions of the left and the right vertices of $U$. Then,

(1) $U$ is called a I-unit of $(\alpha ; \beta)$ if there exists some $i(0 \leq i \leq l)$ such that

$$
\begin{aligned}
& \alpha_{i}^{*}(r) \leq a^{\prime}<a \leq \beta_{i+1}(r), \quad \text { if } U \subset S_{+}, \\
& \alpha_{i}(-r) \leq a<a^{\prime} \leq \beta_{i+1}^{*}(-r), \quad \text { if } U \subset S_{-} .
\end{aligned}
$$

(2) $U$ is called a II-unit of $(\alpha ; \beta)$ if there exists some $i(0 \leq i \leq l)$ such that

$$
\begin{aligned}
& \beta_{i+1}(r) \leq a<a^{\prime} \leq \alpha_{i}^{*}(r), \quad \text { if } U \subset S_{+}, \\
& \beta_{i+1}^{*}(-r) \leq a<a^{\prime} \leq \alpha_{i}(-r), \quad \text { if } U \subset S_{-} .
\end{aligned}
$$

Here, we set $\beta_{l+1}(r)=\beta_{l+1}^{*}(-r)=-\infty$ and $\alpha_{0}(-r)=\alpha_{0}^{*}(r)=+\infty$. Furthermore, a II-unit $U$ of ( $\alpha ; \beta)$ is called a boundary II-unit if (4.5) holds for $i=0, l$, or $r=n$.

For a I-unit, actually (4.4) does not hold for $i=0, l$. Also, it does not hold for $r=n$ if $\lambda / \mu$ satisfies the positivity condition (4.2), by Lemma 4.2.

The dual $U^{*}$ of a unit $U$ is its image by the dual map (4.1). Let $U$ and $U^{\prime}$ be units. If the left or the right vertex of $U$ is also a vertex of $U^{\prime}$, then we say that $U$ and $U^{\prime}$ are adjacent and write $U \diamond U^{\prime}$. It immediately follows from the definition that

\section{Lemma 4.4}

(1) A unit $U$ is a I-unit (resp. a II-unit) if and only if the dual $U^{*}$ is a I-unit (resp. a II-unit).

(2) No unit is simultaneously a I- and II-unit.

(3) If $U$ is a I-unit and $U^{\prime}$ is a II-unit, then $U$ and $U^{\prime}$ are not adjacent.

Fix $(\alpha ; \beta) \in \mathcal{H}(\lambda / \mu)$. Let $\mathcal{U}_{\mathrm{I}}$ be the set of all I-units of $(\alpha ; \beta)$, and let $\tilde{\mathcal{U}}_{\mathrm{I}}:=$ $\bigcup_{U \in \mathcal{U}_{\mathrm{I}}} U$, where the union is taken for $U$ as a subset of $S_{+} \sqcup S_{-}$. Let $\sim$ be the 


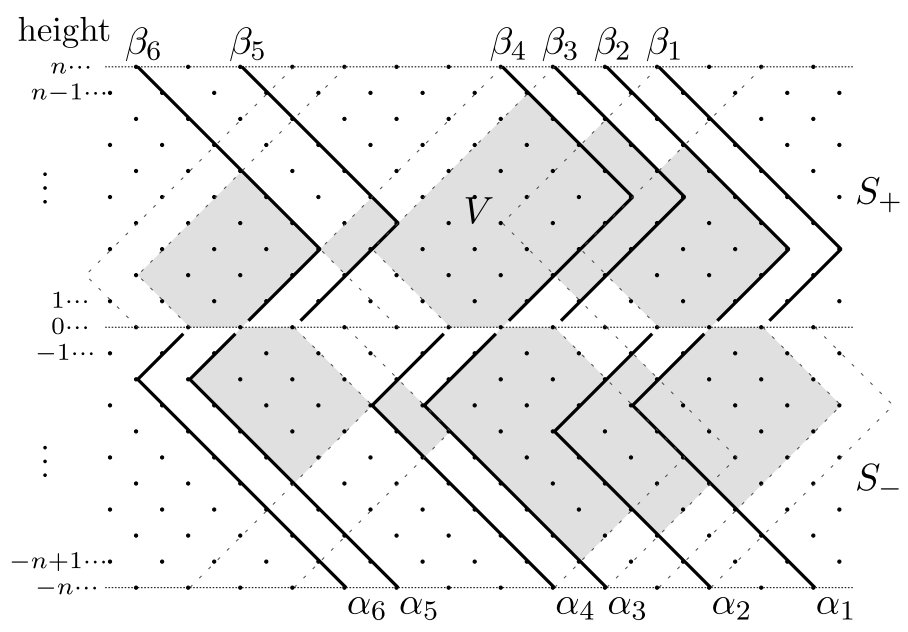

Fig. 3 The undotted lines represent $\alpha_{i}$ 's and $\beta_{i}$ 's while the dotted lines represent their duals, $\alpha_{i}^{*}$ 's and $\beta_{i}^{*}$ 's. The shaded area represents a I-region $V$

equivalence relation in $\mathcal{U}_{\mathrm{I}}$ generated by the relation $\diamond$, and $[U]$ be its equivalence class of $U \in \mathcal{U}_{\mathrm{I}}$. We call $\bigcup_{U^{\prime} \in[U]} U^{\prime}$ a connected component of $\tilde{\mathcal{U}}_{\mathrm{I}}$. For II-units, $\mathcal{U}_{\mathrm{II}}$, $\tilde{\mathcal{U}}_{\text {II }}$ and its connected component are defined similarly.

Definition 4.5 Let $\lambda / \mu$ be a skew diagram satisfying the positivity condition (4.2), and let $(\alpha ; \beta) \in \mathcal{H}(\lambda / \mu)$.

(1) A connected component $V$ of $\tilde{\mathcal{U}}_{\mathrm{I}}$ is called a I-region of $(\alpha ; \beta)$ if it contains at least one I-unit of height 0 .

(2) A connected component $V$ of $\tilde{\mathcal{U}}_{\text {II }}$ is called a II-region of $(\alpha ; \beta)$ if it satisfies the following conditions:

(i) $V$ contains at least one II-unit of height 0.

(ii) $V$ does not contain any boundary II-unit.

See Fig. 3 for an example.

Proposition 4.6 If $V$ is a I- or II-region, then $V^{*}=V$, where for a union of units $V=\bigcup U_{i}$, we define $V^{*}=\bigcup U_{i}^{*}$.

Proof We remark that if two units are adjacent, then their duals are also adjacent. It follows that, for any I-unit $U \subset V, U \sim U_{0} \diamond U_{0}^{*} \sim U^{*}$ holds, where $U_{0}$ is any I-unit $U \subset V$ of height 0 . Therefore, $U^{*} \subset V$.

For any $(\alpha ; \beta) \in \mathcal{H}(\lambda / \mu)$, let $V$ be any I- or II-region of $(\alpha ; \beta)$. Let $\alpha_{i}^{\prime}$ be the lower path obtained from $\alpha_{i}$ by replacing the part $\alpha_{i} \cap V$ with $\beta_{i+1}^{*} \cap V$, and let $\beta_{i}^{\prime}$ be the upper path obtained from $\beta_{i}$ by replacing the part $\beta_{i} \cap V$ with $\alpha_{i-1}^{*} \cap V$. Set $\varepsilon_{V}(\alpha ; \beta):=\left(\alpha_{1}^{\prime}, \ldots, \alpha_{l}^{\prime} ; \beta_{1}^{\prime}, \ldots, \beta_{l}^{\prime}\right)$. See Fig. 4 for an example. 


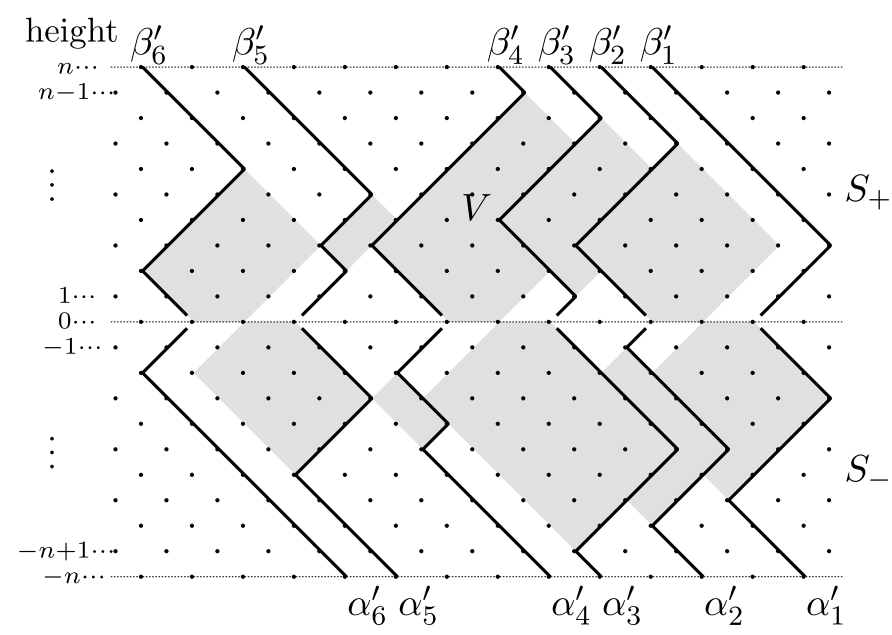

Fig. 4 The tuple $\left(\alpha^{\prime} ; \beta^{\prime}\right):=\varepsilon_{V}(\alpha ; \beta)$ for $(\alpha ; \beta)$ with respect to $V$ in Fig. 3

Proposition 4.7 Let $\lambda / \mu$ be a skew diagram satisfying the positivity condition (4.2). Then, for any $(\alpha ; \beta) \in \mathcal{H}(\lambda / \mu)$, we have

(1) For any I- or II-region $V$ of $(\alpha ; \beta), \varepsilon_{V}(\alpha ; \beta) \in \mathcal{H}(\lambda / \mu)$.

(2) For any I-region $V$ of $(\alpha ; \beta), V$ is a II-region of $\varepsilon_{V}(\alpha ; \beta)$.

(3) For any II-region $V$ of $(\alpha ; \beta), V$ is a I-region of $\varepsilon_{V}(\alpha ; \beta)$.

Proof We give a proof when $V$ is a I-region.

(1) Set $\left(\alpha^{\prime} ; \beta^{\prime}\right):=\varepsilon_{V}(\alpha ; \beta)$. First, since $V$ does not contain any unit of height $\pm n$, we have $\alpha_{i}^{\prime}(-n)=\alpha_{i}(-n)=\frac{n}{2}+\mu_{i}^{\prime}+1-i$ and $\beta_{i}^{\prime}(n)=\beta_{i}(n)=\frac{n}{2}+\lambda_{i}^{\prime}+1-i$. Secondly, let us prove that $\left(\alpha^{\prime} ; \beta^{\prime}\right)$ is nonintersecting. Suppose, for example, if $\left(\alpha_{i}^{\prime}, \alpha_{i+1}^{\prime}\right)$ is intersecting at a point $w$, then it implies that $\left(\alpha_{i}, \beta_{i+2}^{*}\right)$ is intersecting at $w$. Set $-r=\mathrm{ht}(w)$. Since $\alpha_{i}(-r)=\beta_{i+2}^{*}(-r)<\beta_{i+1}^{*}(-r)$, the unit $U \not \subset V$ whose left vertex is $w$ is a I-unit. On the other hand, the unit $U^{\prime}$ whose right vertex is $w$ is in $V$. This contradicts to the fact that $V$ is a connected component of $\tilde{\mathcal{U}}_{\mathrm{I}}$.

(2) It is obvious that a unit in $V$ is a II-unit of $\left(\alpha^{\prime} ; \beta^{\prime}\right)$, and $U \sim U^{\prime}$ for any two units $U, U^{\prime} \subset V$. Assume that there exist some II-unit $U^{\prime \prime} \not \subset V$ of $\left(\alpha^{\prime} ; \beta^{\prime}\right)$ which is adjacent to some $U \subset V$. Since $U^{\prime \prime}$ is a II-unit of $(\alpha ; \beta)$ and $U$ is a I-unit of $(\alpha ; \beta)$, it contradicts to Lemma 4.4 (3). Therefore, $V$ is a connected component of the II-units of $\left(\alpha^{\prime} ; \beta^{\prime}\right)$.

We call the correspondence $(\alpha ; \beta) \mapsto \varepsilon_{V}(\alpha ; \beta)$ the expansion (resp. the folding) with respect to $V$, if $V$ is a I-region (resp. a II-region) of $(\alpha ; \beta)$. We remark that $\varepsilon_{V} \circ \varepsilon_{V}=$ id for any I- or II-region $V$.

Remark 4.8 The expansion and the folding are decomposed into a series of deformations of paths along each unit in $V$. See Fig. 5. This is a key fact in the proof of the weight-preserving property of the maps $\iota_{2}$ in Sect. 4.2 and $\phi$ in Sect. 6. 


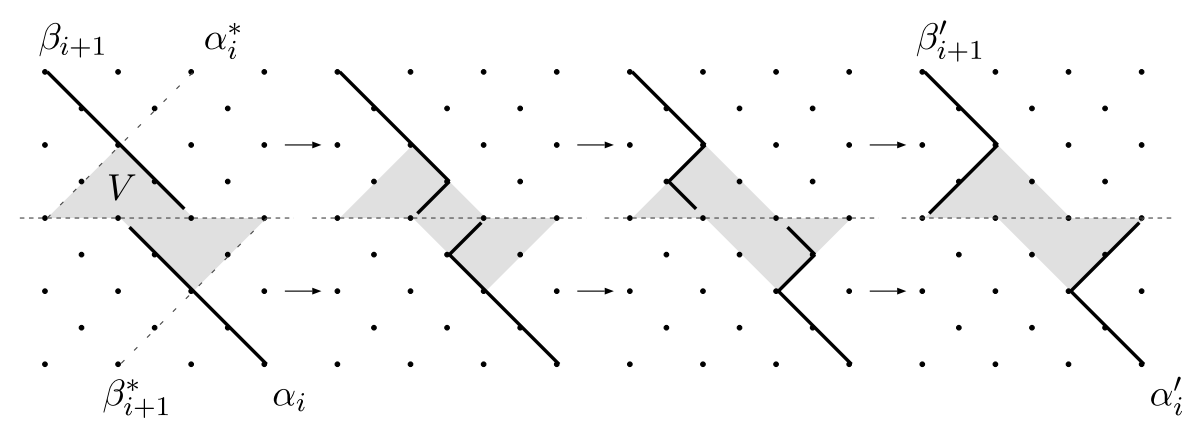

Fig. 5 An example of a procedure of the expansion $(\alpha ; \beta) \mapsto \varepsilon_{V}(\alpha ; \beta)$ with respect to a I-region $V$ at a pair $\left(\alpha_{i}, \beta_{i+1}\right)$, by each unit

\subsection{The second involution and an expression of $\chi_{\lambda / \mu, a}$ by a positive sum}

From now, we assume that $\lambda / \mu$ satisfies the positivity condition (4.2).

Let $p \in P_{1}(\lambda / \mu)$, and let $p_{i}( \pm n)$ be the horizontal position of $p_{i}$ at height $\pm n$. Then $p_{i}(-n)<p_{j}(-n)$ for any $i<j$. We call a pair $\left(p_{i}, p_{j}\right), i<j$ transposed if $p_{i}(n)>p_{j}(n)$.

For each $p \in P_{1}(\lambda / \mu)$, one can uniquely associate $(\alpha ; \beta) \in \mathcal{H}(\lambda / \mu)$ by removing all the E-steps from $p$. We write $\pi(p)$ for $(\alpha ; \beta)$. A I- or II-region of $(\alpha ; \beta)=\pi(p)$ is also called a I- or II-region of $p$.

Let $p \in P_{1}(\lambda / \mu)$ and $(\alpha ; \beta)=\pi(p)$. If $h:=\alpha_{i}(0)-\beta_{i+1}(0)$ is a non-positive number (resp. a positive number), then we call a pair $\left(\alpha_{i}, \beta_{i+1}\right)$ an overlap (resp. a hole). Furthermore, if $h$ is an even number (resp. an odd number), then we say that $\left(\alpha_{i}, \beta_{i+1}\right)$ is even (resp.odd). Using that no triple $\left(p_{i}, p_{j}, p_{k}\right)$ exists for $p \in P_{1}(\lambda / \mu)$ which is intersecting at a point, we have

Lemma 4.9 Let $(\alpha ; \beta)=\pi(p)$ for $p \in P_{1}(\lambda / \mu)$. Then, for any $i$,

(1) $\left(\alpha_{i}, \beta_{i+1}\right)$ is an odd overlap if and only if $\left(p_{i}, p_{j}\right)$ is a specially intersecting, non-transposed pair for some $j>i$.

(2) $\left(\alpha_{i}, \beta_{i+1}\right)$ is an even overlap if and only if $\left(p_{i}, p_{j}\right)$ is a transposed pair for some $j>i$.

(3) $\left(\alpha_{i}, \beta_{i+1}\right)$ is a hole if and only if $\left(p_{i}, p_{j}\right)$ is not intersecting for any $j>i$.

Let $p \in P_{1}(\lambda / \mu),(\alpha ; \beta)=\pi(p)$, and $V$ be a I- or II-region of $p$. Then, there exists $p^{\prime} \in P_{1}(\lambda / \mu)$ such that

$$
\varepsilon_{V}(\alpha ; \beta)=\pi\left(p^{\prime}\right)
$$

It is constructed from $p$ as follows, which is well-defined by Lemma 4.9:

A. The case of a I-region $V$. For any $i$, replace $\left(\alpha_{i}, \beta_{i+1}\right)$ in $p$ with $\left(\alpha_{i}^{\prime}, \beta_{i+1}^{\prime}\right)$. Furthermore, for any $i$ such that $\left(\alpha_{i}, \beta_{i+1}\right)$ is an overlap and intersects with $V$ at height 0 , remove the E-steps between $\beta_{i+1}^{\prime}(0)$ and $\alpha_{i}^{\prime}(0)$. See Fig. 6. 


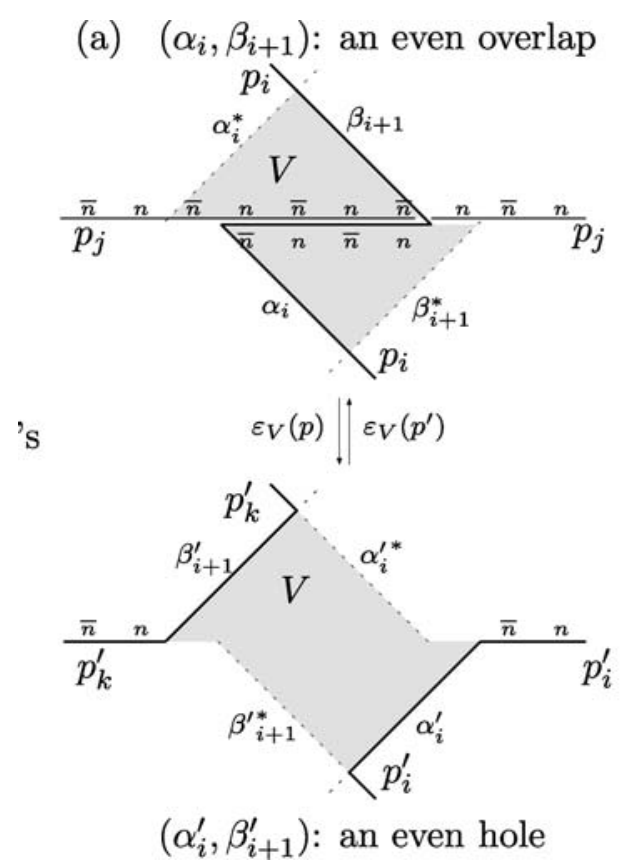

(b) $\left(\alpha_{i}, \beta_{i+1}\right)$ : an odd overlap
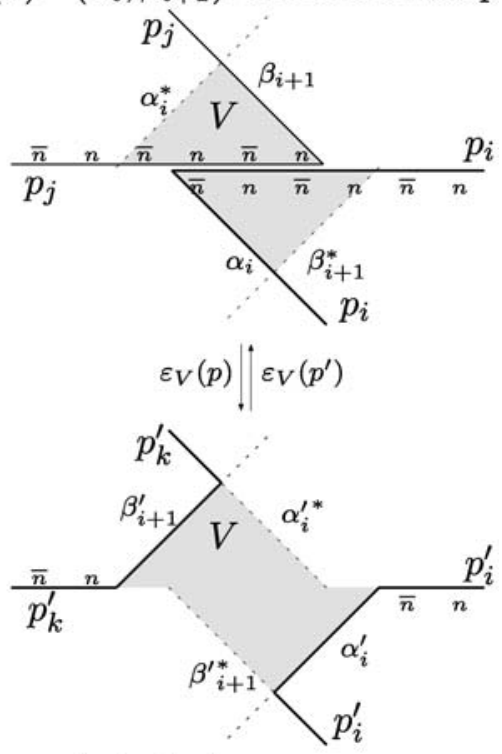

$\left(\alpha_{i}^{\prime}, \beta_{i+1}^{\prime}\right):$ an odd hole

Fig. 6 The deformation $\varepsilon_{V}: p \leftrightarrow p^{\prime}$ with respect to a I-region $V$ of $p$ and a II-region $V$ of $p^{\prime}$

B. The case of a II-region $V$. This is the reverse operation of Case A. Namely, for any $i$, replace $\left(\alpha_{i}, \beta_{i+1}\right)$ in $p$ with $\left(\alpha_{i}^{\prime}, \beta_{i+1}^{\prime}\right)$. Furthermore, for any $i$ such that $\left(\alpha_{i}, \beta_{i+1}\right)$ is a hole and intersects with $V$ at height 0 , then add the E-steps between $\beta_{i+1}^{\prime}(0)$ and $\alpha_{i}^{\prime}(0)$ as in Fig. 6 (a) (for an even hole) and Fig. 6 (b) (for an odd hole) wherein $\left\{\alpha_{i}, \beta_{i+1}, p_{i}, p_{j}\right\}$ and $\left\{\alpha_{i}^{\prime}, \beta_{i+1}^{\prime}, p_{i}^{\prime}, p_{k}^{\prime}\right\}$ are interchanged.

We call the correspondence $p \mapsto p^{\prime}$ the expansion (resp. the folding) of $p$ with respect to a I-region (resp. a II-region) $V$, and write $\varepsilon_{V}(p):=p^{\prime}$.

For any I-region $V$ (resp. II-region $V$ ) of $p \in P_{1}(\lambda / \mu)$ with $(\alpha ; \beta)=\pi(p)$, we set

$$
n(V):=\#\left\{\begin{array}{l}
i \mid \begin{array}{l}
\left(\alpha_{i}, \beta_{i+1}\right) \text { is an even overlap (resp. an even hole) } \\
\text { which intersects with } V \text { at height } 0
\end{array}
\end{array}\right\}
$$

Let $V$ be a I- or II-region of $p \in P_{1}(\lambda / \mu)$. By Lemma 4.9, $n(V)$ is equal to the number of the transposed pairs $\left(p_{i}, p_{j}\right)$ in $p$ which intersect with $V$ at height 0 . Moreover, since the expansion (resp. the folding) $p \mapsto \varepsilon_{V}(p)$ is a deformation that 'resolves' all the transposed pairs (resp. transposes all the even holes) in $p$ which intersect with $V$ at height 0 , we have

Lemma 4.10 Let $p \in P_{1}(\lambda / \mu)$ and $V$ be a I- or II-region of $p$. Then,

$$
(-1)^{\varepsilon_{V}(p)}=(-1)^{n(V)} \cdot(-1)^{p} .
$$

Definition 4.11 We say that a I- or II-region $V$ is even (resp. odd) if $n(V)$ is even (resp. odd). 
Let $P_{\text {odd }}(\lambda / \mu)$ be the set of all $p \in P_{1}(\lambda / \mu)$ which have at least one odd I- or II-region of $p$. We can define an involution

$$
\iota_{2}: P_{\text {odd }}(\lambda / \mu) \rightarrow P_{\text {odd }}(\lambda / \mu)
$$

as follows: Let $V$ be the unique odd I- or II-region of $p \in P_{\text {odd }}(\lambda / \mu)$ such that the value $\max \{\mathrm{hp}(w) \mid w \in V, \operatorname{ht}(w)=0\}$ is greatest among all the odd I- or II-regions of $p$, and set $\iota_{2}(p)=\varepsilon_{V}(p)$. Then we have

Proposition 4.12 The map $\iota_{2}: P_{\mathrm{odd}}(\lambda / \mu) \rightarrow P_{\mathrm{odd}}(\lambda / \mu)$ is a weight-preserving, sign-reversing involution.

Proof The map $\iota_{2}$ is an involution because $\varepsilon_{V} \circ \varepsilon_{V}=\mathrm{id}$, and sign-reversing by Lemma 4.10. We prove that $\iota_{2}$ is weight-preserving in the case where $p \mapsto p^{\prime}:=$ $\iota_{2}(p)$ is an expansion with respect to a I-region $V$ of $p$. Let $(\alpha ; \beta)=\pi(p)$, and we decompose the weights $z_{a}^{p}$ and $z_{a}^{p^{\prime}}$ in (3.4) into two parts as $z_{a}^{p}=H E$ and $z_{a}^{p}=H^{\prime} E^{\prime}$ where $H$ and $H^{\prime}$ are the factors from the $e$-labeling on $(\alpha ; \beta)$ and $\left(\alpha^{\prime} ; \beta^{\prime}\right)$, while $E$ and $E^{\prime}$ are the ones from the $e$-labeling on the height 0 part (the E-steps) of $p$ and $p^{\prime}$. By Remark 4.8, we have $H^{\prime}=H \delta$, where

$$
\delta:=\prod_{U \subset V: \text { unit }} \delta(U),
$$

and, for any unit $U \subset V$ in $S_{ \pm}$of height $\pm r$ with left vertex $(x, y)$,

$$
\delta(U):= \begin{cases}z_{\overline{n-r}, a-2 x} / z_{\overline{n-r+1}}, a-2 x & \text { if } r \neq 0 \text { and } U \subset S_{+}, \\ z_{n-r, a-2 x} / z_{n-r+1, a-2 x}, & \text { if } r \neq 0 \text { and } U \subset S_{-}, \\ z_{\bar{n}, a-2 x}, & \text { if } r=0 \text { and } U \subset S_{+}, \\ z_{n, a-2 x}, & \text { if } r=0 \text { and } U \subset S_{-} .\end{cases}
$$

Using the relations in (2.2), we have $\delta(U) \cdot \delta\left(U^{*}\right)=1$ for any $U$ whose height is not 0 . Therefore, combining $\delta(U)$ for all the I-units in $V$, we obtain

$$
\delta=\prod_{\substack{U \subset V: \text { unit } \\ \operatorname{ht}(U)=0}} \delta(U)=\prod_{i=1}^{l-1}\left(\prod_{k=\alpha_{i}^{*}(0)}^{\beta_{i+1}(0)-1} z_{\bar{n}, a-2 k} \prod_{k=\alpha_{i}(0)}^{\beta_{i+1}^{*}(0)-1} z_{n, a-2 k}\right)
$$

See Fig. 6. On the other hand, we have $E^{\prime}=E \delta^{-1}$, and therefore, we obtain $z_{a}^{\iota_{2}(p)}=$ $H^{\prime} E^{\prime}=H E=z_{a}^{p}$.

It follows from Proposition 4.12 that the contributions of $P_{\text {odd }}(\lambda / \mu)$ to the sum (3.6) cancel each other.

Let $P_{2}(\lambda / \mu):=P_{1}(\lambda / \mu) \backslash P_{\text {odd }}(\lambda / \mu)$, i.e., the set of all $p \in P_{1}(\lambda / \mu)$ which satisfy the following conditions:

(i) $p$ does not have any ordinarily intersecting pair $\left(p_{i}, p_{j}\right)$.

(ii) $p$ does not have any odd I- or II-region. 
Every $p \in P_{2}(\lambda / \mu)$ has an even number of transposed pairs, which implies that $(-1)^{p}=1$. Thus, the sum (3.6) reduces to a positive sum, and we have

Theorem 4.13 For any skew diagram $\lambda / \mu$ satisfying the positivity condition (4.2), we have

$$
\chi_{\lambda / \mu, a}=\sum_{p \in P_{2}(\lambda / \mu)} z_{a}^{p} .
$$

\section{The folding map and a tableaux description}

In this section, we give a tableaux description of $\chi_{\lambda / \mu, a}$. Namely, the sum (4.7) is translated into the one over a set of the tableaux of shape $\lambda / \mu$ which satisfy certain conditions called the horizontal, vertical, and extra rules.

\subsection{The folding map}

Since a path $p \in P_{2}(\lambda / \mu)$ in (4.7) might have (an even number of) transposed pairs $\left(p_{i}, p_{j}\right)$, the sum (4.7) cannot be translated into a tableaux description yet. Therefore, we introduce another set of paths as follows.

Let $P(\lambda / \mu)$ be the set of all $p=\left(p_{1}, \ldots, p_{l}\right) \in \mathfrak{P}\left(\mathrm{id} ; u_{\mu}, v_{\lambda}\right)$ such that

(i) $p$ does not have any ordinarily intersecting adjacent pair $\left(p_{i}, p_{i+1}\right)$.

(ii) $p$ does not have any odd II-region.

Here, an odd II-region of $p \in P(\lambda / \mu)$ is defined in the same way as that of $p \in$ $P_{1}(\lambda / \mu)$. The following fact is not so trivial.

Proposition 5.1 There exists a weight-preserving bijection

$$
\phi: P_{2}(\lambda / \mu) \rightarrow P(\lambda / \mu)
$$

The map $\phi$ is called the folding map. Roughly speaking, it is an iterated application of (some generalization of) the folding in Sect. 4. The construction of $\phi$ is the most technical part of the paper. We provide the details in Sect. 6. Admitting Proposition 5.1, we immediately have

Theorem 5.2 For any skew diagram $\lambda / \mu$ satisfying the positivity condition (4.2), we have

$$
\chi_{\lambda / \mu, a}=\sum_{p \in P(\lambda / \mu)} z_{a}^{p}
$$

\subsection{Tableaux description}

Define a partial order in $I$ in (2.1) by

$$
1 \prec 2 \prec \cdots \prec n-1 \prec \frac{n}{n} \prec \overline{n-1} \prec \cdots \prec \overline{2} \prec \overline{1} .
$$


A tableau $T$ of shape $\lambda / \mu$ is the skew diagram $\lambda / \mu$ with each box filled by one entry of $I$. For a tableau $T$ and $a \in \mathbb{C}$, we define the weight of $T$ as

$$
z_{a}^{T}=\prod_{(i, j) \in \lambda / \mu} z_{T(i, j), a+2(j-i)},
$$

where $T(i, j)$ is the entry of $T$ at $(i, j)$.

Definition 5.3 A tableau $T$ (of shape $\lambda / \mu$ ) is called an HV-tableau if it satisfies the following conditions:

(H) horizontal rule $T(i, j) \preceq T(i, j+1)$ or $(T(i, j), T(i, j+1))=(n, \bar{n})$.

(V) vertical rule $T(i, j) \nsucceq T(i+1, j)$.

We denote the set of all HV-tableaux of shape $\lambda / \mu$ by $\operatorname{Tab}_{\mathrm{HV}}(\lambda / \mu)$.

Remark 5.4 The configuration $(T(i, j), T(i, j+1))=(n, \bar{n})$ is prohibited later by another rule. See Remark 5.11.

Let $P_{\mathrm{HV}}(\lambda / \mu)$ be the set of all $p \in \mathfrak{P}\left(\mathrm{id} ; u_{\mu}, v_{\lambda}\right)$ which do not have any ordinarily intersecting adjacent pair $\left(p_{i}, p_{i+1}\right)$. With any $p \in P_{\mathrm{HV}}(\lambda / \mu)$, we associate a tableau $T$ of shape $\lambda / \mu$ as follows: For any $j=1, \ldots, l$, let $E\left(p_{j}\right)=\left\{s_{i_{1}}, s_{i_{2}}, \ldots, s_{i_{m}}\right\}\left(i_{1}<\right.$ $i_{2}<\cdots<i_{m}$ ) be the set defined as in (3.1), and set

$$
T\left(\mu_{j}^{\prime}+k, j\right)=L_{a}^{1}\left(s_{i_{k}}\right), \quad k=1, \ldots, m,
$$

where $L_{a}^{1}$ is the first component of the $e$-labeling (3.2). It is easy to see that $T$ satisfies the vertical rule $(\mathbf{V})$ because of the definition of the $e$-labeling of $p_{j}$, and satisfies the horizontal rule $(\mathbf{H})$ because $p$ does not have any ordinarily intersecting adjacent pair. Therefore, if we set $\mathcal{T}_{\mathrm{v}}: p \mapsto T$, we have

Proposition 5.5 The map

$$
\mathcal{T}_{\mathrm{v}}: P_{\mathrm{HV}}(\lambda / \mu) \rightarrow \operatorname{Tab}_{\mathrm{HV}}(\lambda / \mu)
$$

is a weight-preserving bijection.

Let $\operatorname{Tab}(\lambda / \mu):=\mathcal{T}_{\mathrm{v}}(P(\lambda / \mu))$. In other words, $\operatorname{Tab}(\lambda / \mu)$ is the set of all the tableaux $T$ which satisfy $(\mathbf{H}),(\mathbf{V})$, and the following extra rule:

(E) The corresponding $p=\mathcal{T}_{\mathrm{v}}^{-1}(T)$ does not have any odd II-region.

By Theorem 5.2 and Proposition 5.5, we obtain a tableaux description of $\chi_{\lambda / \mu, a}$, which is the main result of the paper.

Theorem 5.6 For any skew diagram $\lambda / \mu$ satisfying the positivity condition (4.2), we have

$$
\chi_{\lambda / \mu, a}=\sum_{T \in \operatorname{Tab}(\lambda / \mu)} z_{a}^{T} .
$$




\subsection{Extra rule in terms of tableau}

It is straightforward to translate the extra rule $(\mathbf{E})$ into tableau language. We only give the result here.

Fix an HV-tableau $T$. For any $a_{1}, \ldots, a_{m} \in I$, let $C\left(a_{1}, \ldots, a_{m}\right)$ be a configuration in $T$ as follows:

$$
\begin{array}{|c|}
\hline a_{1} \\
\hline a_{2} \\
\hline \vdots \\
\hline a_{m} \\
\hline
\end{array}
$$

If $1 \preceq a_{1} \prec \cdots \prec a_{m} \preceq n$, then we call it an L-configuration. If $\bar{n} \preceq a_{1} \prec \cdots \prec a_{m} \preceq \overline{1}$, then we call it a $U$-configuration. Note that an L-configuration corresponds to a part of a lower path, while a U-configuration corresponds to a part of an upper path under the map $\mathcal{T}_{\mathrm{v}}$.

Let $(L, U)$ be a pair of an L-configuration $L=C\left(a_{1}, \ldots, a_{s}\right)$ in the $j$ th column and a U-configuration $U=C\left(\overline{b_{t}}, \ldots, \overline{b_{1}}\right)$ in the $(j+1)$ th column. We call it an $L U$ configuration of $T$ if it satisfies one of the following two conditions:

Condition 1. LU-configuration of type 1. $(L, U)$ has the form

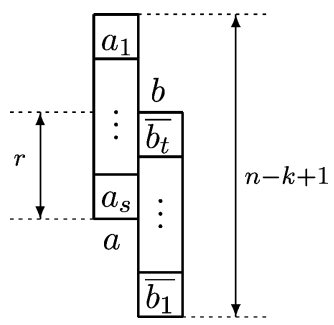

for some $k$ and $r$ with $1 \leq k \leq n, 1 \leq r \leq \min \{s, t\}, n-k+1=s+t-r$, and

$$
\begin{aligned}
a_{1}=k, & \overline{b_{1}}=\bar{k}, \\
a \succeq \bar{n} \text { if } a \text { exists, } & b \preceq n \text { if } b \text { exists, } \\
a_{i+1} \preceq b_{i}^{\prime}, \quad\left(1 \leq i \leq t^{\prime}\right), & \overline{b_{i+1}} \succeq \overline{a_{i}^{\prime}}, \quad\left(1 \leq i \leq s^{\prime}\right),
\end{aligned}
$$

where $a_{1}^{\prime} \prec \cdots \prec a_{s^{\prime}}^{\prime}\left(s^{\prime}:=t-r\right)$ and $b_{1}^{\prime} \prec \cdots \prec b_{t^{\prime}}^{\prime}\left(t^{\prime}:=s-r\right)$ are defined as

$$
\begin{aligned}
\left\{a_{1}, \ldots, a_{s}\right\} \sqcup\left\{a_{1}^{\prime}, \ldots, a_{s^{\prime}}^{\prime}\right\} & =\{k, k+1, \ldots, n\}, \\
\left\{\overline{b_{1}}, \ldots, \overline{b_{t}}\right\} \sqcup\left\{\overline{b_{1}^{\prime}}, \ldots, \overline{b_{t^{\prime}}^{\prime}}\right\} & =\{\bar{k}, \overline{k+1}, \ldots, \bar{n}\} .
\end{aligned}
$$

See Fig. 7 for the corresponding part in the paths. In particular, if $r$ is odd, then we say that $(L, U)$ is odd. 


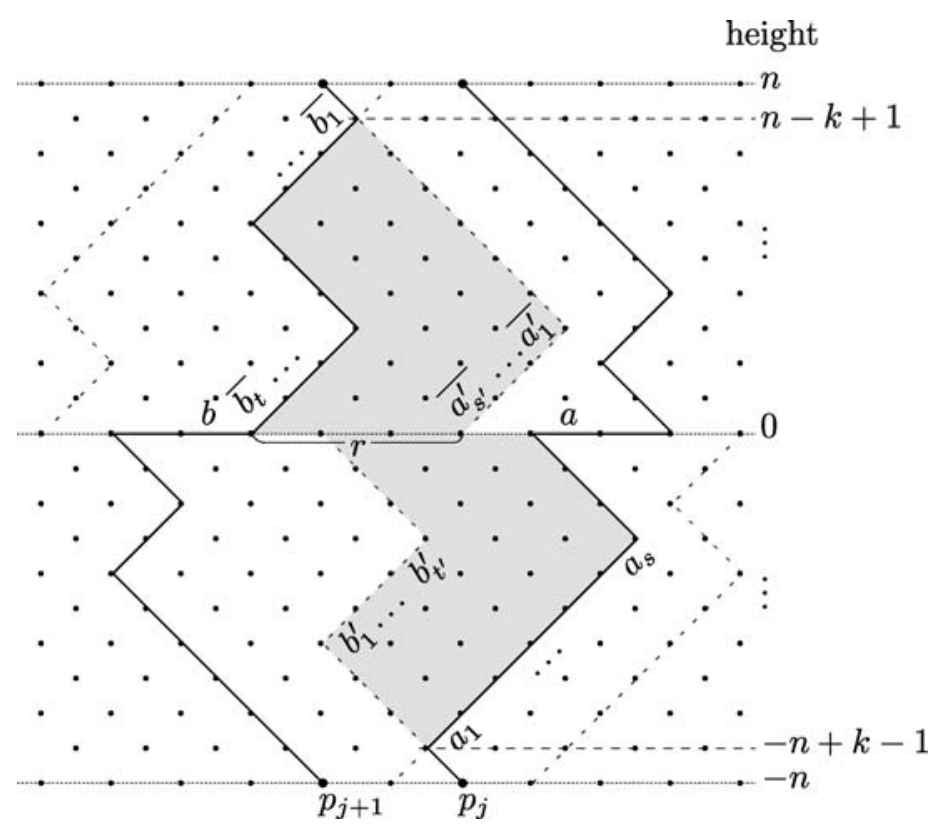

Fig. 7 An example of adjacent paths $\left(p_{j}, p_{j+1}\right)$ such that a part of it corresponds to an LU-configuration of type 1 as in (5.3)

Condition 2. LU-configuration of type 2. $(L, U)$ has the form

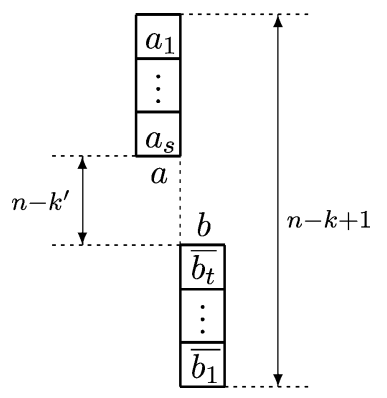

for some $k$ and $k^{\prime}$ with $1 \leq k<k^{\prime} \leq n, n-k+1=n-k^{\prime}+s+t$, and

$$
\begin{array}{clrl}
a_{1}=k, \quad \overline{b_{1}}=\bar{k}, \quad a_{s^{\prime}}^{\prime}=k^{\prime}, & \overline{b_{t^{\prime}}^{\prime}}=\overline{k^{\prime}}, \quad a \npreceq k^{\prime}, \quad b \nsucceq \overline{k^{\prime}}, \\
a_{i+1} \preceq b_{i}^{\prime}, \quad(1 \leq i<s), & \overline{b_{i+1}} \succeq \overline{a_{i}^{\prime}}, \quad(1 \leq i<t),
\end{array}
$$

where $a_{1}^{\prime} \prec \cdots \prec a_{s^{\prime}}^{\prime}\left(s^{\prime}:=t\right)$ and $b_{1}^{\prime} \prec \cdots \prec b_{t^{\prime}}^{\prime}\left(t^{\prime}:=s\right)$ are defined by

$$
\begin{aligned}
& \left\{a_{1}, \ldots, a_{s}\right\} \sqcup\left\{a_{1}^{\prime}, \ldots, a_{s^{\prime}}^{\prime}\right\}=\left\{k, k+1, \ldots, k^{\prime}\right\}, \\
& \left\{\overline{b_{1}}, \ldots, \overline{b_{t}}\right\} \sqcup\left\{\overline{b_{1}^{\prime}}, \ldots, \overline{b_{t^{\prime}}^{\prime}}\right\}=\left\{\bar{k}, \overline{k+1}, \ldots, \overline{k^{\prime}}\right\} .
\end{aligned}
$$

See Fig. 8 for the corresponding part in the paths. 
Fig. 8 An example of adjacent paths $\left(p_{j}, p_{j+1}\right)$ such that a part of it corresponds to an LU-configuration of type 2 as in (5.8)

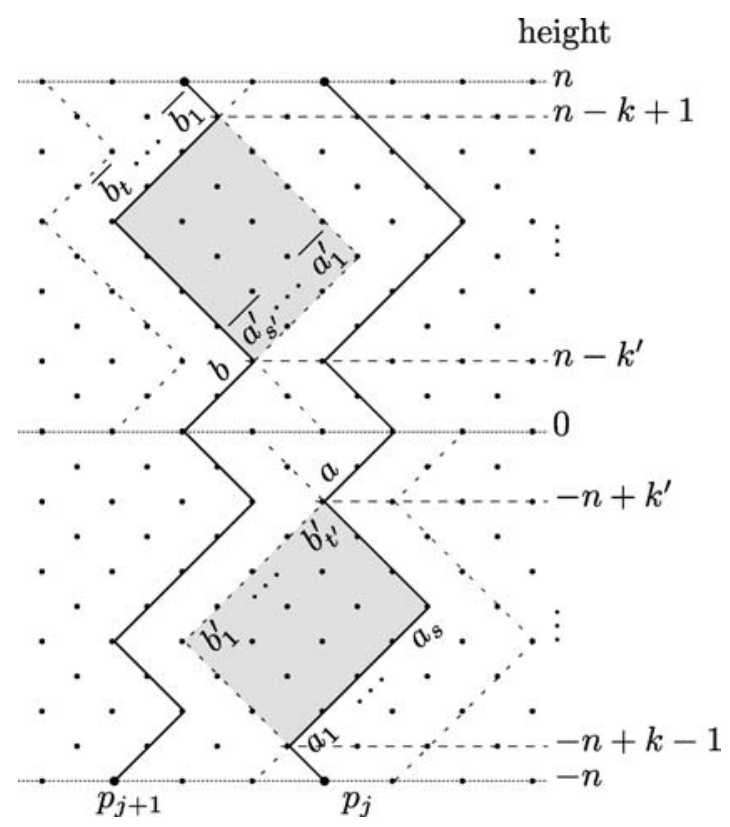

We say that an $L$-configuration $L=C\left(a_{1}, \ldots, a_{m}\right)$ in the $j$ th column of $T$ is boundary if $a_{1}=T\left(\mu_{j}^{\prime}+1, j\right)$, i.e., if $a_{1}$ is at the top of the $j$ th column, and $m$ is the largest number such that $L \cap L^{\prime}=\emptyset$ for any $L U$-configuration $\left(L^{\prime}, U^{\prime}\right)$. Similarly, a $U$-configuration $U=C\left(a_{1}, \ldots, a_{m}\right)$ in the $j$ th column of $T$ is boundary if $a_{m}=$ $T\left(\lambda_{j}^{\prime}, j\right)$, i.e., if $a_{m}$ is at the bottom of the $j$ th column, and $m$ is the largest number such that $U \cap U^{\prime}=\emptyset$ for any $L U$-configuration $\left(L^{\prime}, U^{\prime}\right)$.

Let $(L, U)=\left(C\left(a_{1}, \ldots, a_{s}\right), C\left(\overline{b_{t}}, \ldots, \overline{b_{1}}\right)\right)$ be an LU-configuration, and set $a_{1}^{\prime} \prec$ $\cdots \prec a_{s^{\prime}}^{\prime}$ and $b_{1}^{\prime} \prec \cdots \prec b_{t^{\prime}}^{\prime}$ as in (5.7) (resp. as in (5.11)) if $(L, U)$ is of type 1 (resp. of type 2). We say that an L-configuration $L^{\prime}$ is right-adjacent to $(L, U)$ if $L^{\prime}$ is in the right-next column to $L$; furthermore, there exists some pair of an entry $e$ of $L^{\prime}$ and an entry $a_{i}$ of $L$ such that $e$ is right-next to $a_{i}$ and $e \prec b_{i}^{\prime}$. Similarly, we say that a U-configuration $U^{\prime}$ is left-adjacent to $(L, U)$ if $U^{\prime}$ is in the left-next column to $U$; furthermore, there exists some pair of an entry $e$ of $U^{\prime}$ and an entry $\overline{b_{i}}$ of $U$ such that $e$ is left-next to $\overline{b_{i}}$ and $e \succ \overline{a_{i}^{\prime}}$. Then, we say that an LU-configuration $\left(L^{\prime}, U^{\prime}\right)$ is adjacent to $(L, U)$ if one of the following conditions is satisfied, and write it by $(L, U) \diamond\left(L^{\prime}, U^{\prime}\right)$ :

(i) $L^{\prime}$ is right-adjacent to $(L, U)$.

(ii) $L$ is right-adjacent to $\left(L^{\prime}, U^{\prime}\right)$.

(iii) $U^{\prime}$ is left-adjacent to $(L, U)$.

(iv) $U$ is left-adjacent to $\left(L^{\prime}, U^{\prime}\right)$.

For any tableau $T$, let $\mathcal{L U}(T)$ be the set of all LU-configurations of $T$. Then, the adjacent relation $\diamond$ of the LU-configurations generates an equivalence relation $\sim$ in $\mathcal{L U}(T)$. 
Definition 5.7 For any $(L, U) \in \mathcal{L U}(T)$, let $[(L, U)] \subset \mathcal{L U}(T)$ be the equivalence class of $(L, U)$ with respect to $\sim$, and let $R=R(L, U):=\bigcup_{\left(L^{\prime}, U^{\prime}\right) \in[(L, U)]}\left(L^{\prime}, U^{\prime}\right)$ be the corresponding configuration in $T$. We call $R$ a II-region of $T$, if the following conditions is satisfied:

(1) No boundary L-configuration $L$ is right-adjacent to $L^{\prime}$ for any LU-configuration $\left(L^{\prime}, U^{\prime}\right)$ in $R$.

(2) No boundary U-configuration $U$ is left-adjacent to $U^{\prime}$ for any LU-configuration $\left(L^{\prime}, U^{\prime}\right)$ in $R$.

Moreover, we say that $R$ is odd if the number of the odd type 1 LU-configurations in $R$ is odd.

Then, an odd II-region of $T=\mathcal{T}_{\mathrm{v}}(p)$ corresponds to an odd II-region of $p$, and therefore, Theorem 5.6 is rewritten as follows:

Theorem 5.8 For any skew diagram $\lambda / \mu$ satisfying the positivity condition (4.2), we have

$$
\chi_{\lambda / \mu, a}=\sum_{T \in \operatorname{Tab}(\lambda / \mu)} z_{a}^{T},
$$

where $\operatorname{Tab}(\lambda / \mu)$ is the set of all the tableaux of shape $\lambda / \mu$ which satisfy $(\mathbf{H}),(\mathbf{V})$, and the following extra rule $\left(\mathbf{E}^{\prime}\right)$ :

( $\left.\mathbf{E}^{\prime}\right) \quad T$ does not have any odd II-region.

\subsection{Explicit list of odd II-regions}

Let us give an explicit list of all the possible odd II-regions for $\lambda / \mu$ of at most two columns or of at most three rows.

Example 5.9 Let $\lambda / \mu$ be a skew diagram of two columns satisfying the positivity condition (4.2). In this case, an odd II-region of $T \in \operatorname{Tab}_{\mathrm{HV}}(\lambda / \mu)$ is nothing but an odd type 1 LU-configuration without any boundary L-configuration $L$ which is right-adjacent to it, or any boundary $\mathrm{U}$-configuration $U$ which is left-adjacent to it. Therefore, the extra rule $\left(\mathbf{E}^{\prime}\right)$ is given as follows:

(E-2C) $T$ does not include any odd type 1 LU-configuration as

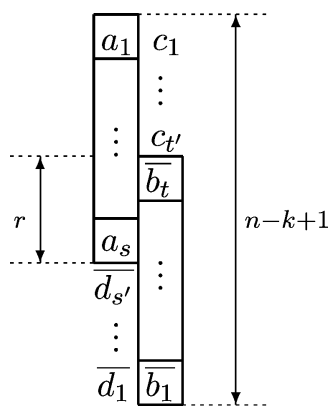


Table 1 The LU-configurations in a tableau in $\operatorname{Tab}(\lambda / \mu)$ for a skew diagram $\lambda / \mu$ of at most three rows. We omit $a$ and $b$ if the inequalities are satisfied by the vertical rule $(\mathbf{V})$

odd type 1

LU-configurations

$(a \succeq \bar{n}, b \preceq n)$ other

LU-configurations

$(a \succeq \bar{n}, b \preceq n$,

$\left.a^{\prime} \succ n-1, b^{\prime} \prec \overline{n-1}\right)$

\begin{tabular}{|c|c|c|c|c|c|c|c|c|c|}
\hline & & & & & & $n-2$ & $\bar{n}$ & $n-2$ & $b$ \\
\hline & & $n-1$ & $b$ & $n-1$ & $\bar{n}$ & $a$ & $\overline{n-1}$ & $n-1$ & $\overline{n-1}$ \\
\hline$n$ & $\bar{n}$ & $n$ & $\overline{n-1}$ & $a$ & $\overline{n-1}$ & & $\overline{n-2}$ & $a$ & $\overline{n-2}$ \\
\hline$n-2$ & & $n-2$ & $b$ & $n-2$ & & $n-2$ & $\bar{n}$ & & \\
\hline$n-1$ & $\bar{n}$ & $n$ & $\overline{n-1}$ & $n-1$ & $b$ & $n-1$ & $\overline{n-1}$ & & \\
\hline$a$ & $\overline{n-2}$ & & $\overline{n-2}$ & $n$ & $\overline{n-2}$ & $n$ & $\overline{n-2}$ & & \\
\hline
\end{tabular}
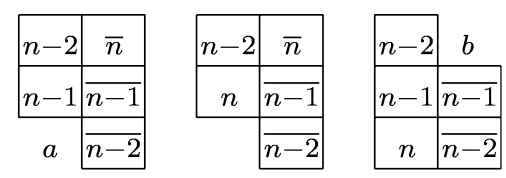

\begin{tabular}{|c|c|}
\cline { 1 - 2 }$n-2$ & \\
\hline$n-1$ & $\bar{n}$ \\
\hline$n$ & $\overline{n-2}$ \\
\hline
\end{tabular}

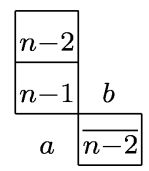

\begin{tabular}{|l|l}
$r n-2$ & \\
$a^{\prime}$ & $b^{\prime}$ \\
& $\overline{n-2}$ \\
&
\end{tabular}

where, if $t^{\prime}=t-r \geq 1$, then $c_{t^{\prime}} \preceq n$ and $c_{i} \succeq b_{i}^{\prime}$ for any $i=1, \ldots, t^{\prime}$, and if $s^{\prime}=$ $s-r \geq 1$, then $d_{s^{\prime}} \succeq \bar{n}$ and $\overline{d_{i}} \preceq \overline{a_{i}^{\prime}}$ for any $i=1, \ldots, s^{\prime}$.

Example 5.10 Let $\lambda$ be a Young diagram of one row. In this case, the odd II-region of $T \in \operatorname{Tab}_{\mathrm{HV}}(\lambda)$ is the configuration \begin{tabular}{|l|l|}
\hline$n$ & $\bar{n}$
\end{tabular} , and therefore, the extra rule $\left(\mathbf{E}^{\prime}\right)$ is given as follows:

(E-1R) $\quad T$ does not include \begin{tabular}{|l|l|}
\hline$n$ & $\bar{n}$ \\
\hline
\end{tabular}

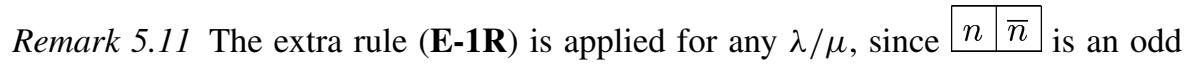
II-region of $T \in \operatorname{Tab}_{\mathrm{HV}}(\lambda / \mu)$.

Example 5.12 Let $\lambda / \mu$ be a skew diagram of two rows, i.e., $\lambda_{1}^{\prime}=2$. Since an odd II-region of $T \in \operatorname{Tab}_{\mathrm{HV}}(\lambda / \mu)$ is a combination of the LU-configurations of at most two rows in Table 1 with one odd type 1 LU-configuration. Therefore, the extra rule $\left(\mathbf{E}^{\prime}\right)$ is given by $(\mathbf{E}-\mathbf{1 R})$ and the following condition:

(E-2R) $T$ does not include
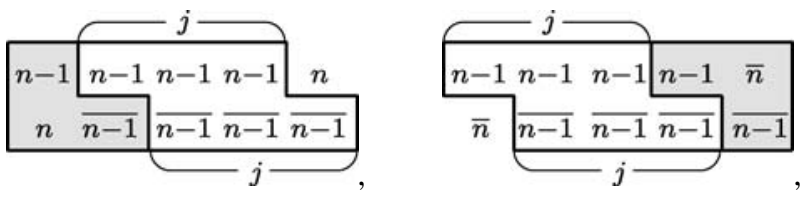

where $j \geq 0$. 
This rule appears for the proposed crystals of the representations of two-row rectangle [21].

Example 5.13 Let $\lambda / \mu$ be a skew diagram of three rows, i.e., $\lambda_{1}^{\prime}=3$. Since an odd II-region of $T \in \operatorname{Tab}_{\mathrm{HV}}(\lambda / \mu)$ is a combination of the LU-configurations of at most three rows in Table 1 with one or three odd type 1 LU-configurations, the extra rule $\left(\mathbf{E}^{\prime}\right)$ for a tableau $T \in \operatorname{Tab}_{\mathrm{HV}}(\lambda / \mu)$ is given by $(\mathbf{E}-\mathbf{1 R}),(\mathbf{E}-\mathbf{2 R})$, and the following condition:

(E-3R) $\quad T$ does not include

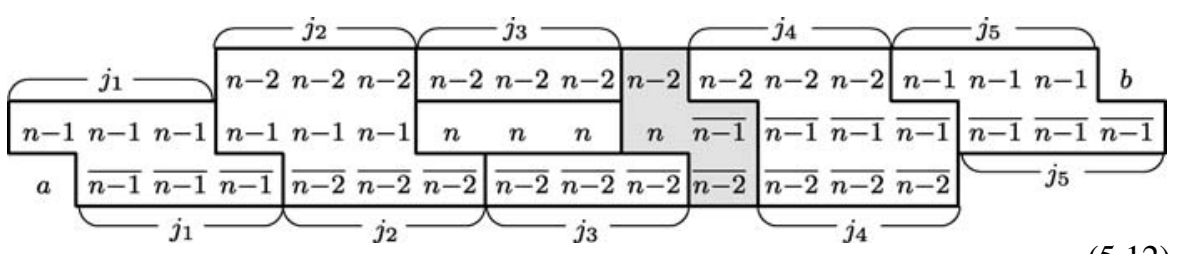

where $j_{i} \geq 0, j_{1}=0$ if $j_{2}=0$, and

$$
(a, b)= \begin{cases}(\bar{n}, n),(n, \bar{n}), & \text { if } j_{2} \neq 0 \\ (\bar{n}, n),(\overline{n-1}, n) & \text { if } j_{2}=0\end{cases}
$$

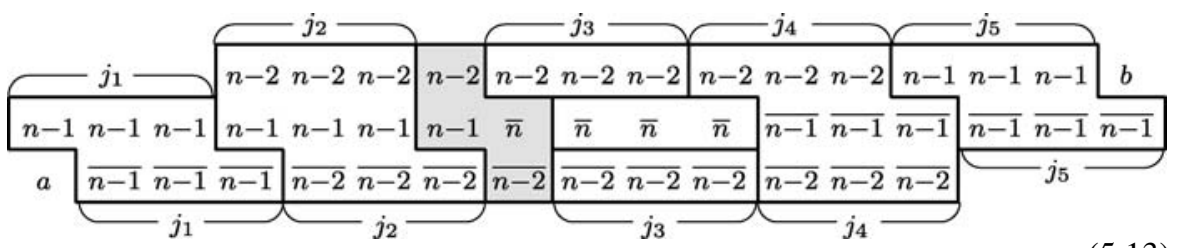

where $j_{i} \geq 0, j_{5}=0$ if $j_{4}=0$, and

$$
(a, b)= \begin{cases}(\bar{n}, n),(n, \bar{n}), & \text { if } j_{4} \neq 0, \\ (\bar{n}, n-1),(\bar{n}, n), & \text { if } j_{4}=0,\end{cases}
$$

and

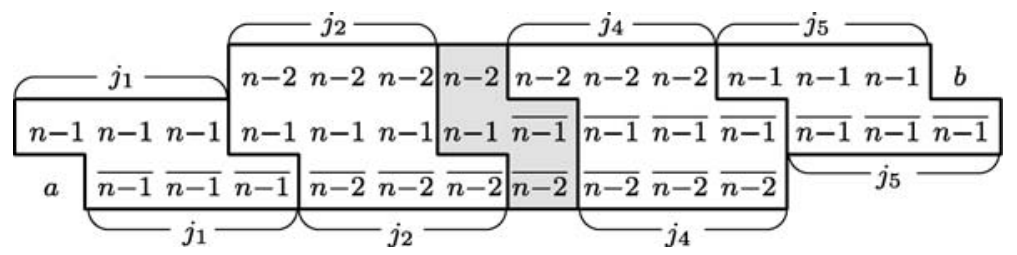

where $j_{i} \geq 0$ and $(a, b)=(n, \bar{n})$ or $(\bar{n}, n)$.

The odd II-regions (5.12), (5.13), and (5.14) include three (resp. one) odd type 1 LU-configurations if $(a, b)=(n, \bar{n}), j_{1}+j_{2} \geq 1$, and $j_{4}+j_{5} \geq 1$ are satisfied (resp. otherwise). 


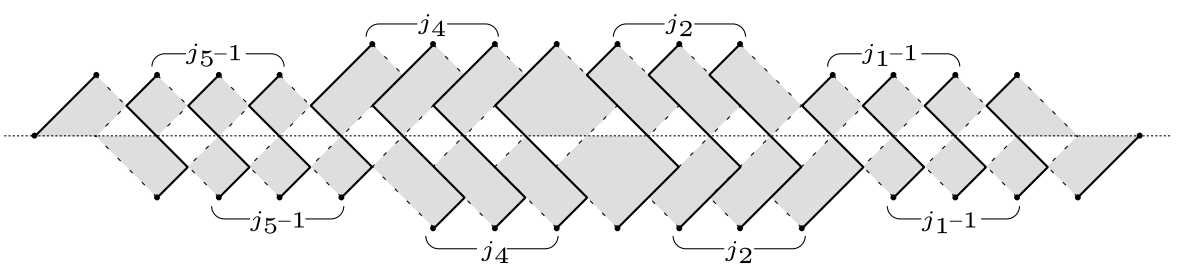

Fig. 9 The odd II-region corresponding to the rule (5.14) for $(a, b)=(n, \bar{n}), j_{1}, j_{5} \neq 0$

As mentioned in the introduction, though these rules look rather complicated, they are more easily recognizable in the path picture. For example, the rule (5.14) for $(a, b)=(n, \bar{n}), j_{1}, j_{5} \neq 0$ corresponds to the odd II-region in Fig. 9.

\section{Construction of $\phi$ and proof of Proposition 5.1}

In this section, we construct the folding map $\phi: P_{2}(\lambda / \mu) \rightarrow P(\lambda / \mu)$ in Proposition 5.1, which is a key to derive the tableaux description (see Theorem 5.6).

\section{$6.1 k$-expansion and $k$-folding}

To define $\phi$, we introduce the $k$-expansion and the $k$-folding, which are generalizations of the expansion and the folding in Sect. 4.1. The original corresponds to $k=1$. We also generalize related notions.

Definition 6.1 Let $(\alpha ; \beta) \in \mathcal{H}(\lambda / \mu)$. For any unit $U \subset S_{ \pm}$, let $\pm r=\operatorname{ht}(U)$ and let $a$ and $a^{\prime}=a+1$ be the horizontal positions of the left and the right vertices of $U$. Then, for any $k=1, \ldots, l-1$,

(1) $U$ is called a $\mathrm{I}_{k}$-unit of $(\alpha ; \beta)$ if there exists some $i(0 \leq i \leq l)$ such that

$$
\begin{aligned}
& \alpha_{i}^{*}(r) \leq a<a^{\prime} \leq \beta_{i+k}(r), \quad \text { if } U \subset S_{+}, \\
& \alpha_{i}(-r) \leq a<a^{\prime} \leq \beta_{i+k}^{*}(-r), \quad \text { if } U \subset S_{-} .
\end{aligned}
$$

(2) $U$ is called a $\mathrm{II}_{k}$-unit of $(\alpha ; \beta)$ if there exists some $i(0 \leq i \leq l)$ such that

$$
\begin{aligned}
& \beta_{i+k}(r) \leq a<a^{\prime} \leq \alpha_{i}^{*}(r), \quad \text { if } U \subset S_{+}, \\
& \beta_{i+k}^{*}(-r) \leq a<a^{\prime} \leq \alpha_{i}(-r), \quad \text { if } U \subset S_{-} .
\end{aligned}
$$

Here, we set $\beta_{i}(r)=\beta_{i}^{*}(-r)=-\infty$ for $i \geq l+1$ and $\alpha_{i}(-r)=\alpha_{i}^{*}(r)=+\infty$ for $i \leq 0$. Furthermore, a $\mathrm{II}_{k}$-unit $U$ of $(\alpha ; \beta)$ is called a boundary $\mathrm{II}_{k}$-unit if (6.2) holds for $i=0, i \geq l-k+1$, or $r=n$.

As in the $k=1$ case, actually (6.1) does not hold for $i=0$ or $i \geq l-k+1$. Also it does not hold for $r=n$ if $\lambda / \mu$ satisfies the positivity condition. 
As Lemma 4.4, it is easy to see that

\section{Lemma 6.2}

(1) $U$ is a $\mathrm{I}_{k}$-unit (resp. a $\mathrm{II}_{k}$-unit) if and only if $U^{*}$ is a $\mathrm{I}_{k}$-unit (resp. a $\mathrm{II}_{k}$-unit).

(2) No unit is simultaneously $a \mathrm{I}_{k^{-}}$and $\mathrm{II}_{k^{\prime}}$-unit for any $k$ and $k^{\prime}$ such that $k+1 \geq k^{\prime}$.

(3) If $U$ is a $\mathrm{I}_{k}$-unit and $U^{\prime}$ is a $\mathrm{II}_{k^{\prime}}$-unit for any $k \geq k^{\prime}$, then $U$ and $U^{\prime}$ are not adjacent.

(4) The set of all the $\mathrm{I}_{k}$-units and that of all the $\mathrm{II}_{k+1}$-units are complementary to each other in $S_{+} \sqcup S_{-}$.

We define $\mathcal{U}_{\mathrm{I}_{k}}$ and $\tilde{\mathcal{U}}_{\mathrm{I}_{k}}$ (resp. $\mathcal{U}_{\mathrm{II}_{k}}$ and $\tilde{\mathcal{U}}_{\mathrm{II}_{k}}$ ) for $\mathrm{I}_{k}$-units (resp. $\mathrm{II}_{k}$-units) of $(\alpha ; \beta)$ and a connected component of $\tilde{\mathcal{U}}_{\mathrm{I}_{k}}$ and $\tilde{\mathcal{U}}_{\mathrm{II}_{k}}$ similarly as in the $k=1$ case.

Definition 6.3 Let $\lambda / \mu$ be a skew diagram satisfying the positivity condition (4.2), and let $(\alpha ; \beta) \in \mathcal{H}(\lambda / \mu)$.

(1) A connected component $V$ of $\tilde{\mathcal{U}}_{\mathrm{I}_{k}}$ is called a $\mathrm{I}_{k}$-region of $(\alpha ; \beta)$ if it contains at least one $\mathrm{I}_{k}$-unit of height 0 .

(2) A connected component $V$ of $\tilde{\mathcal{U}}_{\mathrm{II}_{k}}$ is called a $\mathrm{II}_{k}$-region of $(\alpha ; \beta)$ if it satisfies the following conditions:

(i) $V$ contains at least one $\mathrm{I}_{k}$-unit of height 0 .

(ii) $V$ does not contain any boundary $\mathrm{II}_{k}$-unit.

As Proposition 4.6, we have

Proposition 6.4 If $V$ is a $\mathrm{I}_{k}$ - or $\mathrm{II}_{k}$-region of $(\alpha ; \beta)$, then $V^{*}=V$.

If $U$ is a $\mathrm{I}_{k}$-unit of $(\alpha ; \beta)$, then it is also a $\mathrm{I}_{k^{\prime}}$-unit $(\alpha ; \beta)$ for any $1 \leq k^{\prime} \leq k$, while if $U$ is a $\mathrm{II}_{k}$-unit of $(\alpha ; \beta)$, then it is also a $\mathrm{II}_{k^{\prime}}$-unit of $(\alpha ; \beta)$ for any $k \leq k^{\prime} \leq l-1$. Then, it follows that

\section{Lemma 6.5}

(1) If $V$ is a $\mathrm{I}_{k}$-region and $V^{\prime}$ is a $\mathrm{I}_{k^{\prime}}$-region for $k^{\prime} \geq k$, then $V^{\prime} \subset V$ or $V \cap V^{\prime}=\emptyset$.

(2) If $V$ is a $\mathrm{II}_{k}$-region and $V^{\prime}$ is a $\mathrm{II}_{k^{\prime}}$-region for $k^{\prime} \leq k$, then $V^{\prime} \subset V$ or $V \cap V^{\prime}=\emptyset$.

For any $(\alpha ; \beta) \in \mathcal{H}(\lambda / \mu)$, let $V$ be any $\mathrm{I}_{k}-$ or $\mathrm{II}_{k}$-region of $(\alpha ; \beta)$. Let $\alpha_{i}^{\prime}$ be the lower path obtained from $\alpha_{i}$ by replacing the part $\alpha_{i} \cap V$ with $\beta_{i+k}^{*} \cap V$, and let $\beta_{i}^{\prime}$ be the upper path obtained from $\beta_{i}$ by replacing the part $\beta_{i} \cap V$ with $\alpha_{i-k}^{*} \cap V$. Set $\varepsilon_{V}^{k}(\alpha ; \beta):=\left(\alpha_{1}^{\prime}, \ldots, \alpha_{l}^{\prime} ; \beta_{1}^{\prime}, \ldots, \beta_{l}^{\prime}\right)$. Then, Proposition 4.7 is generalized as follows:

Proposition 6.6 Let $\lambda / \mu$ be a skew diagram satisfying the positivity condition (4.2). Then, for any $(\alpha ; \beta) \in \mathcal{H}(\lambda / \mu)$, we have

(1) For any $\mathrm{I}_{k}$ - or $\mathrm{II}_{k}$-region $V$ of $(\alpha ; \beta), \varepsilon_{V}^{k}(\alpha ; \beta) \in \mathcal{H}(\lambda / \mu)$.

(2) If $V$ is a $\mathrm{I}_{k}$-region and $V^{\prime} \subset V$ is a $\mathrm{I}_{r}$-region of $(\alpha ; \beta)$ for $r \geq k$, then $V^{\prime}$ is a $\mathrm{II}_{2 k-r}$-region of $\varepsilon_{V}^{k}(\alpha ; \beta)$. 
(3) If $V$ is a $\mathrm{II}_{k}$-region and $V^{\prime} \subset V$ is a $\mathrm{II}_{r}$-region of $(\alpha$; $\beta)$ for $r \leq k$, then $V^{\prime}$ is a $\mathrm{I}_{2 k-r}$-region of $\varepsilon_{V}^{k}(\alpha ; \beta)$.

Set $\left(\alpha^{\prime} ; \beta^{\prime}\right)=\varepsilon_{V}^{k}(\alpha ; \beta)$ for any $\mathrm{I}_{k}$ - or $\mathrm{II}_{k}$-region $V$. The following equalities are useful:

$$
\begin{aligned}
& \alpha_{i}^{\prime}(0)= \begin{cases}\beta_{i+k}^{*}(0), & \text { if } \beta_{i+r} \text { intersects with } V \text { at hight } 0, \\
\alpha_{i}(0), & \text { otherwise, }\end{cases} \\
& \beta_{i}^{\prime}(0)= \begin{cases}\alpha_{i-k}^{*}(0), & \text { if } \alpha_{i-k} \text { intersects with } V \text { at height } 0, \\
\beta_{i}(0), & \text { otherwise. }\end{cases}
\end{aligned}
$$

We call the correspondence $(\alpha ; \beta) \mapsto \varepsilon_{V}^{k}(\alpha ; \beta)$ the $k$-expansion (resp. the $k$-folding) with respect to $V$, if $V$ is a $\mathrm{I}_{k}$-region (resp. a $\mathrm{II}_{k}$-region). As the $k=1$ case, we have $\varepsilon_{V}^{k} \circ \varepsilon_{V}^{k}=$ id for any $V$.

Let $(\alpha ; \beta) \in \mathcal{H}(\lambda / \mu)$. If $h:=\alpha_{i}(0)-\beta_{i+k}(0)$ is a non-positive number (resp. a positive number), then we call a pair $\left(\alpha_{i}, \beta_{i+k}\right)$ a $k$-overlap (resp. a $k$-hole). Furthermore, if $h$ is an even number (resp. an odd number), then $\left(\alpha_{i}, \beta_{i+k}\right)$ is called even (resp. odd).

For any $\mathrm{I}_{k}$-region $V$ (resp. $\mathrm{II}_{k}$-region $V$ ) of $(\alpha ; \beta) \in \mathcal{H}(\lambda / \mu)$, let $n(V)$ be the number of the even $k$-overlaps (resp. the even $k$-holes) which intersect with $V$ at height 0 .

Definition 6.7 $\mathrm{A}_{k^{-}}$or $\mathrm{II}_{k}$-region $V$ is called even (resp. odd) if $n(V)$ is even (resp. odd).

\subsection{Outline of construction}

In this subsection, we give the outline of the construction of the folding map $\phi$ whose existence is admitted in Sect. 5. Proofs of Propositions 6.9-6.12 below will be given in the following sections.

Let $t_{0}$ be the minimal number that satisfies $2^{t_{0}}>l$. For any $t=1,2, \ldots, t_{0}$, we define

$$
\begin{aligned}
& Q_{t}(\lambda / \mu):=\left\{(\alpha ; \beta) \in \mathcal{H}(\lambda / \mu) \mid(\alpha ; \beta) \text { satisfies Conditions }(1)_{t}-(6)_{t}\right\}, \\
& \hat{Q}_{t}(\lambda / \mu):=\left\{(\alpha ; \beta) \in \mathcal{H}(\lambda / \mu) \mid(\alpha ; \beta) \text { satisfies Conditions }(1)_{t}-(7)_{t}\right\},
\end{aligned}
$$

where Conditions $(1)_{t}-(7)_{t}$ are given as follows:

(1) $\alpha_{i}(0) \leq \beta_{i}(0)$ for any $i=1, \ldots, l$.

(2) $)_{t}(\alpha ; \beta)$ does not contain any odd $\mathrm{II}_{1}$-region.

(3) $t_{t}(\alpha ; \beta)$ does not contain any odd $\mathrm{I}_{2^{t}-1}$-region.

$(4)_{t}(\alpha ; \beta)$ does not contain any $2^{t}$-overlap.

$(5)_{t}$ If $t \geq 2$, then $(\alpha ; \beta)$ contains at least one $2^{t-1}$-overlap.

(6) ${ }_{t} s\left(\alpha_{i}\right) \equiv m_{t}\left(\alpha_{i}\right)$ and $s\left(\beta_{i}\right) \equiv m_{t}\left(\beta_{i}\right)$, where 


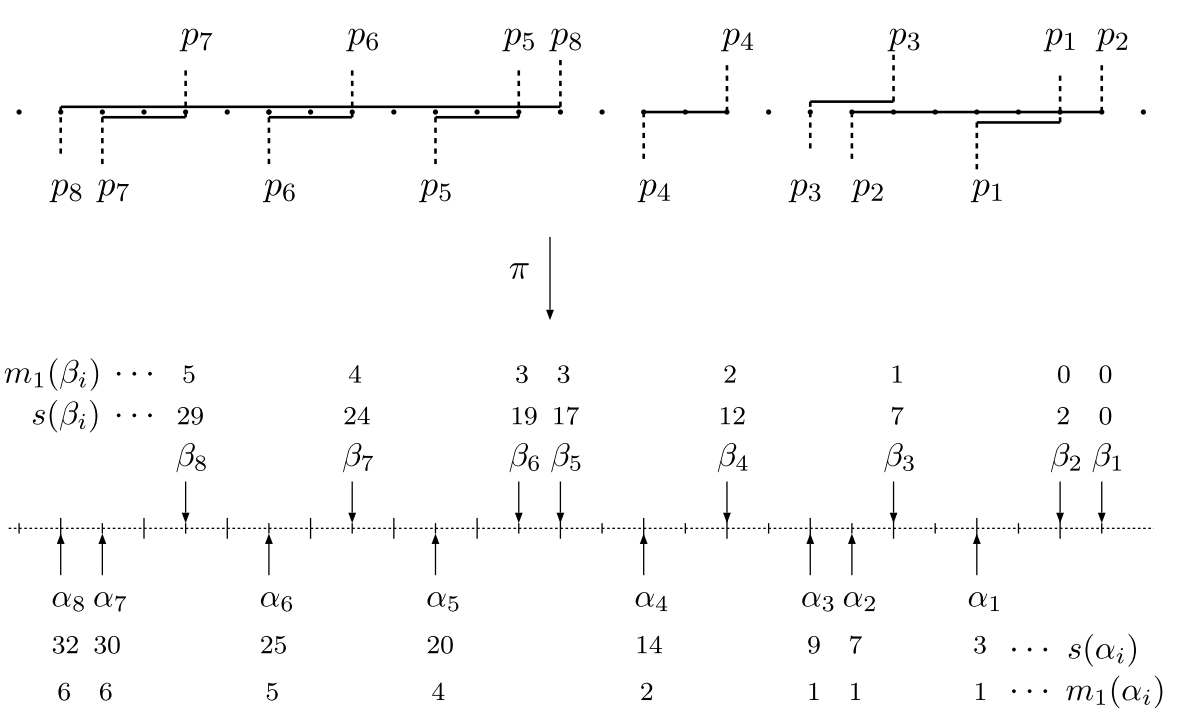

Fig. 10 An example of $s\left(\alpha_{i}\right), s\left(\beta_{i}\right), m\left(\alpha_{i}\right)$, and $m\left(\beta_{i}\right)$ in the condition $(6)_{t}$ of $Q_{t}(\lambda / \mu)$ for $t=1$

$$
\begin{aligned}
s\left(\alpha_{i}\right):= & \alpha_{i}(0)-\beta_{1}(0)+i-1, \\
s\left(\beta_{i}\right):= & \beta_{i}(0)-\beta_{1}(0)+i-1, \\
m_{t}\left(\alpha_{i}\right):= & \#\left\{j \mid j \leq i,\left(\alpha_{j}, \beta_{j+2^{t}-1}\right) \text { is an even }\left(2^{t}-1\right) \text {-overlap }\right\} \\
& +\#\left\{j \mid j<i,\left(\alpha_{j}, \beta_{j+1}\right) \text { is an even 1-hole }\right\}, \\
m_{t}\left(\beta_{i}\right):= & \#\left\{j \mid j<i,\left(\alpha_{j-2^{t}+1}, \beta_{j}\right) \text { is an even }\left(2^{t}-1\right) \text {-overlap }\right\} \\
& +\#\left\{j \mid j \leq i,\left(\alpha_{j-1}, \beta_{j}\right) \text { is an even 1-hole }\right\} .
\end{aligned}
$$

Here and the rest part of this section, $\equiv$ denotes the congruence modulo 2 . See Fig. 10.

$(7)_{t}(\alpha ; \beta)$ has at least one even $\left(2^{t}-1\right)$-overlap (Then, it has at least two, because of Condition $(3)_{t}$ ).

Remark 6.8 If $t=t_{0}$, then Conditions (3) $t$ and (4) $t$ are void. Also, $\hat{Q}_{t_{0}}(\lambda / \mu)=\emptyset$, because $(7)_{t}$ is not satisfied for any $(\alpha ; \beta) \in \mathcal{H}(\lambda / \mu)$.

Now, the folding map $\phi: P_{2}(\lambda / \mu) \rightarrow P(\lambda / \mu)$ is constructed as follows: For any $p \in P_{2}(\lambda / \mu)$ or any $p \in P(\lambda / \mu)$, one can associate $\pi(p):=(\alpha ; \beta) \in \mathcal{H}(\lambda / \mu)$ by removing all the E-steps from $p$. Then, we have

Proposition 6.9 The map $\pi$ gives a bijection $P_{2}(\lambda / \mu) \rightarrow Q_{1}(\lambda / \mu)$.

The following claim is the main part of the construction of $\phi$ :

Proposition 6.10 There exists a bijection $\varphi_{t}: \hat{Q}_{t}(\lambda / \mu) \rightarrow Q_{t+1}(\lambda / \mu)$ for any $t=$ $1, \ldots, t_{0}-1$. 
The map $\varphi_{t}$ is defined by using the $2^{t}$-folding. See (6.8). Applying the bijections $\varphi_{1}, \varphi_{2}, \ldots$ repeatedly, we obtain a bijection $\varphi: Q_{1}(\lambda / \mu) \rightarrow R(\lambda / \mu)$, where

$$
R(\lambda / \mu):=\bigsqcup_{t=1}^{t_{0}}\left(Q_{t}(\lambda / \mu)-\hat{Q}_{t}(\lambda / \mu)\right) .
$$

Furthermore, we have

Proposition 6.11 The map $\pi$ gives a bijection $P(\lambda / \mu) \rightarrow R(\lambda / \mu)$.

Thanks to Propositions 6.9-6.11, we now have a bijection $\phi$ by the commutative diagram,

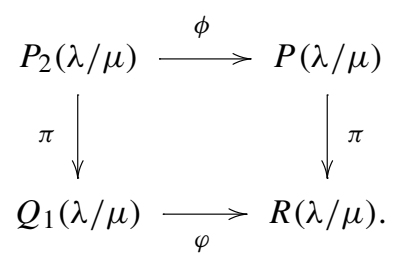

Then, Proposition 5.1 follows from

Proposition 6.12 The map $\phi$ is weight-preserving.

\subsection{Proof of Proposition 6.9}

We use the following lemma which immediately follows by the definition of $s\left(\alpha_{i}\right)$ and $s\left(\beta_{i}\right)$ :

Lemma 6.13 For any $i=1, \ldots, l$,

(1) $\alpha_{i}(0)-\beta_{j}(0)$ is even if and only if $s\left(\alpha_{i}\right)-i \equiv s\left(\beta_{j}\right)-j$.

(2) $\alpha_{i}(0)-\alpha_{j}(0)$ is even if and only if $s\left(\alpha_{i}\right)-i \equiv s\left(\alpha_{j}\right)-j$.

(3) $\beta_{i}(0)-\beta_{j}(0)$ is even if and only if $s\left(\beta_{i}\right)-i \equiv s\left(\beta_{j}\right)-j$.

First, we prove that

$$
\pi\left(P_{2}(\lambda / \mu)\right) \subset Q_{1}(\lambda / \mu) .
$$

Let $p \in P_{2}(\lambda / \mu)$ and set $(\alpha ; \beta)=\pi(p)$. It holds for any $i=1, \ldots, l$ that

$$
\#\left\{j \mid \alpha_{i}(0) \leq \beta_{j}(0)\right\} \geq i
$$

Therefore, $(1)_{t}$ (for $t=1$ ) is satisfied. By Condition (ii) of $P_{2}(\lambda / \mu),(2)_{t}$ and (3) are satisfied, and by Condition (i), (4) $t$ is satisfied.

We prove Condition (6) for $\beta_{i}$ by induction with respect to $i$. The proof for $\alpha_{i}$ is similar. For $i=1$, we have $s\left(\beta_{1}\right)=m_{1}\left(\beta_{1}\right)=0$. Assume $s\left(\beta_{i-1}\right) \equiv m_{1}\left(\beta_{i-1}\right)$. The following four cases should be considered:

(a) $\left(\alpha_{i-1}, \beta_{i}\right)$ is an even 1-hole, and $\left(\alpha_{i-2}, \beta_{i-1}\right)$ is an even 1-overlap. 
(b) $\left(\alpha_{i-1}, \beta_{i}\right)$ is an even 1-hole, and $\left(\alpha_{i-2}, \beta_{i-1}\right)$ is not an even 1-overlap.

(c) $\left(\alpha_{i-1}, \beta_{i}\right)$ is not an even 1-hole, and $\left(\alpha_{i-2}, \beta_{i-1}\right)$ is an even 1-overlap.

(d) $\left(\alpha_{i-1}, \beta_{i}\right)$ is not an even 1-hole, and $\left(\alpha_{i-2}, \beta_{i-1}\right)$ is not an even 1-overlap.

By the definition of $m_{1}\left(\beta_{i}\right)$, we have $m_{1}\left(\beta_{i}\right)=m_{1}\left(\beta_{i-1}\right)+2$ for (a), $m_{1}\left(\beta_{i-1}\right)+1$ for (b) and (c), and $m_{1}\left(\beta_{i-1}\right)$ for (d). On the other hand, by Condition (i) of $P_{2}(\lambda / \mu)$, we have

Lemma 6.14 $\alpha_{i-1}(0)-\beta_{i-1}(0)$ is odd if and only if one and the only one of $\left(\alpha_{i-2}, \beta_{i-1}\right)$ and $\left(\alpha_{i-1}, \beta_{i}\right)$ is an even overlap.

By Lemma 6.14,

$$
\beta_{i}(0)-\beta_{i-1}(0)=\left(\beta_{i}(0)-\alpha_{i-1}(0)\right)+\left(\alpha_{i-1}(0)-\beta_{i-1}(0)\right)
$$

is odd for (a) and (d), and even for (b) and (d). By the assumption of induction and Lemma 6.13 (3), we obtain $s\left(\beta_{i}\right) \equiv m_{1}\left(\beta_{i}\right)$ in each case.

Next, we define the inverse map $\pi^{-1}: Q_{1}(\lambda / \mu) \rightarrow P_{2}(\lambda / \mu)$. For any $(\alpha ; \beta) \in$ $Q_{1}(\lambda / \mu)$, set $p:=\pi^{-1}(\alpha ; \beta)$ as follows: Let $p_{i}:=\left[\alpha_{i}, \beta_{j}\right]$ denote the path defined by $\alpha_{i}, \beta_{j}$ and the consecutive E-steps from $\alpha_{i}(0)$ to $\beta_{j}(0)$.

Step 1. First, for any $i$ such that $\left(\alpha_{i}, \beta_{i+1}\right)$ is an even 1-overlap, set $p_{i}=\left[\alpha_{i}, \beta_{i+1}\right]$ (see $p_{1}, p_{5}, p_{6}$, and $p_{7}$ in Fig. 10 for examples). Let $\Lambda_{1}$ (resp. $\Lambda_{2}$ ) be the set of all $i$ such that $\alpha_{i}$ (resp. $\beta_{i}$ ) do not form even 1-overlaps.

Step 2. Next, for any $i \in \Lambda_{1} \cap \Lambda_{2}$ such that $h:=\alpha_{i}(0)-\beta_{i}(0)$ is even (and nonpositive by $(1)_{t}$ ), set $p_{i}=\left[\alpha_{i}, \beta_{i}\right]$ (see $p_{3}$ and $p_{4}$ in Fig. 10 for examples). Let $\Lambda_{1}^{\prime} \subset \Lambda_{1}$ (resp. $\Lambda_{2}^{\prime} \subset \Lambda_{2}$ ) be the set of all $i$ such that $h$ are not even.

Step 3. Finally, for any $i \in \Lambda_{1}^{\prime} \cap \Lambda_{2}^{\prime}$, there exists some $k>0$ such that $h^{\prime}:=$ $\alpha_{i}(0)-\beta_{i-k}(0)<0$, by (6.7) and the previous steps. Let $k$ be the minimum of such numbers. Since $\left(\alpha_{i-k^{\prime}}, \beta_{i-k^{\prime}+1}\right)$ for all $1 \leq k^{\prime} \leq k$ is an even 1-overlap, we have $m_{1}\left(\alpha_{i}\right)=m_{t}\left(\beta_{i-k}\right)+k$. Then, by Condition $(6)_{t}, h^{\prime}$ is even by Lemma 6.13 (1). Set $p_{i}=\left[\alpha_{i}, \beta_{i-k}\right]$ (see $p_{2}$ and $p_{8}$ in Fig. 10 for examples).

Next, we prove that $p$ satisfies Condition (i) of $P_{2}(\lambda / \mu)$. Namely, for any intersecting pair $\left(p_{i}, p_{j}\right)(i<j)$, we prove that $\alpha_{i}(0)-\alpha_{j}(0)$ is odd. The following two cases should be considered (see Fig. 10; the other cases do not occur by Condition $\left.(4)_{t}\right)$ :

(A) The case where $p_{i}$ is defined in Step 1 and $p_{j}$ is defined in Step 3. In this case, all $p_{k}(i<k<j)$ are defined in Step 1, by the definition of $p_{j}$. Therefore, $\left(\alpha_{k}, \beta_{k+1}\right)$ for any $i<k<j$ is an even 1-overlap, and we have $m_{1}\left(\alpha_{j}\right)=m_{1}\left(\alpha_{i}\right)+j-i-1$. Thus, $\alpha_{i}(0)-\alpha_{j}(0)$ is odd by Condition $(6)_{t}$ and Lemma $6.13(2)$.

(B) The case where $\left(p_{i}, p_{j}\right)$ is a pair of paths defined in Steps (2) and (3). In this case, $p_{j}=\left[\alpha_{j}, \beta_{i+1}\right]$ and $\left(\alpha_{i}, \beta_{i+1}\right)$ is a 1-overlap (otherwise, it contradicts to $\left.(4)_{t}\right)$. Moreover, $\left(\alpha_{i}, \beta_{i+1}\right)$ is odd (otherwise, $p_{i}$ should be $\left[\alpha_{i}, \beta_{i+1}\right]$ ). Since $\alpha_{j}(0)-\beta_{i+1}(0)$ is even by $p_{j}$, we obtain that $\alpha_{i}(0)-\alpha_{j}(0)$ is odd.

Finally, the facts that $p$ satisfies Condition (ii) of $P_{2}(\lambda / \mu)$ and $\pi^{-1}$ is the inverse of $\pi$ are obvious by construction. 


\subsection{Proof of Proposition 6.11}

Set

$$
m_{\infty}\left(\alpha_{i}\right)=m_{\infty}\left(\beta_{i}\right):=\#\left\{j \mid j \leq i \text { and }\left(\alpha_{j-1}, \beta_{j}\right) \text { is an even 1-hole }\right\}
$$

Then

Lemma 6.15 Let $t=1, \ldots, t_{0}$. For any $(\alpha ; \beta) \in \mathcal{H}(\lambda / \mu),(\alpha ; \beta) \in Q_{t}(\lambda / \mu) \backslash$ $\hat{Q}_{t}(\lambda / \mu)$ if and only if $(\alpha ; \beta)$ satisfies conditions $(1)_{t},(2)_{t},(4)_{t},(5)_{t}$ and the following condition:

$(6)_{t}^{\prime} \quad s\left(\alpha_{i}\right) \equiv s\left(\beta_{i}\right) \equiv m_{\infty}\left(\alpha_{i}\right) \equiv m_{\infty}\left(\beta_{i}\right)$.

Proof $(\Rightarrow)$ If $(\alpha ; \beta) \in Q_{t}(\lambda / \mu) \backslash \hat{Q}_{t}(\lambda / \mu)$, then

$$
m_{t}\left(\alpha_{i}\right)=m_{t}\left(\beta_{i}\right)=m_{\infty}\left(\alpha_{i}\right)=m_{\infty}\left(\beta_{i}\right)
$$

and we obtain $(6)_{t}^{\prime}$.

$(\Leftarrow)$ We show that $(\alpha ; \beta)$ does not have any even $\left(2^{t}-1\right)$-overlap. If a pair $\left(\alpha_{i}, \beta_{i+2^{t}-1}\right)$ is an even $\left(2^{t}-1\right)$-overlap, then $s\left(\alpha_{i}\right) \not \equiv s\left(\beta_{i+2^{t}-1}\right)$ by Lemma $6.13(1)$. On the other hand, there does not exist any 1-hole between $\alpha_{i}$ and $\beta_{i+2^{t}-1}$, which implies $m_{\infty}\left(\alpha_{i}\right)=m_{\infty}\left(\beta_{i+2^{t}-1}\right)$, and it contradicts to $(6)_{t}^{\prime}$. Therefore, $(7)_{t}$ is satisfied, and moreover, we also have $m_{\infty}\left(\alpha_{i}\right)=m_{t}\left(\alpha_{i}\right)$ and $m_{\infty}\left(\beta_{i}\right)=m_{t}\left(\beta_{i}\right)$. Thus, $(6)_{t}$ is satisfied. Finally, $(3)_{t}$ is satisfied because every $\mathrm{I}_{2^{t}-1}$-region $V$ of $(\alpha ; \beta)$ satisfies $n(V)=0$ by the definition of $n(V)$.

By Lemma 6.15, the set $R(\lambda / \mu)$ in (6.4) is described as follows:

$$
R(\lambda / \mu)=\left\{(\alpha ; \beta) \in \mathcal{H}(\lambda / \mu) \mid(\alpha ; \beta) \text { satisfies }(1)_{t},(2)_{t} \text { and }(6)_{t}^{\prime}\right\} .
$$

Next, we prove $\pi(P(\lambda / \mu)) \subset R(\lambda / \mu)$. Conditions $(1)_{t}$ and $(2)_{t}$ are satisfied, similarly as in the proof of (6.6). We prove (6) $t_{t}^{\prime}$. Since $p_{i}=\left[\alpha_{i}, \beta_{i}\right], \alpha_{i}(0)-\beta_{i}(0)$ is even. Thus, $s\left(\alpha_{i}\right) \equiv s\left(\beta_{i}\right)$ by Lemma 6.13 (1). We prove $s\left(\alpha_{i}\right) \equiv m_{\infty}\left(\alpha_{i}\right)$ by induction with respect to $i$. The proof for $s\left(\alpha_{i}\right) \equiv m_{\infty}\left(\beta_{i}\right)$ is similar. For $i=1$, we have $s\left(\beta_{1}\right)=m_{\infty}\left(\alpha_{1}\right)=0$, and we obtain $s\left(\alpha_{1}\right) \equiv s\left(\beta_{1}\right) \equiv m_{\infty}\left(\alpha_{1}\right)$. Assume $s\left(\alpha_{i-1}\right) \equiv m_{\infty}\left(\alpha_{i-1}\right)$. Then the following two cases should be considered:

(a) $\left(\alpha_{i-1}, \beta_{i}\right)$ is an even 1 -hole.

(b) $\left(\alpha_{i-1}, \beta_{i}\right)$ is not an even 1-hole.

By the definition, $m_{\infty}\left(\alpha_{i}\right)=m_{\infty}\left(\alpha_{i-1}\right)+1$ for (a) and $m_{\infty}\left(\alpha_{i-1}\right)$ for (b). On the other hand, we have $s\left(\beta_{i}\right) \not \equiv s\left(\alpha_{i-1}\right)$ for (a), and $s\left(\beta_{i}\right) \equiv s\left(\alpha_{i-1}\right)$ for (b). Therefore, we obtain $s\left(\alpha_{i}\right) \equiv s\left(\beta_{i}\right) \equiv m_{\infty}\left(\alpha_{i}\right)$ in each case.

Finally, we define the inverse map $\pi^{-1}: R(\lambda / \mu) \rightarrow P(\lambda / \mu)$ by $p:=\pi^{-1}(\alpha ; \beta)$, $p_{i}:=\left[\alpha_{i}, \beta_{i}\right](i=1, \ldots, l)$ for any $(\alpha ; \beta) \in R(\lambda / \mu)$. The path $p_{i}$ is well defined, because of (6) ${ }_{t}^{\prime}$ and Lemma 6.13 (1). If $\left(p_{i}, p_{i+1}\right)$ is ordinarily intersecting, then we have $s\left(\alpha_{i}\right) \not \equiv s\left(\alpha_{i+1}\right)$ by Lemma 6.13 (2) and $m_{\infty}\left(\alpha_{i}\right)=m_{\infty}\left(\alpha_{i+1}\right)$, which contradicts to (6) $)_{t}^{\prime}$. Therefore, $p$ satisfies Condition (i) of $P(\lambda / \mu)$. The facts that $p$ satisfies Condition (ii) of $P(\lambda / \mu)$ and $\pi^{-1}$ is the inverse of $\pi$ are obvious. 


\subsection{Definition of $\varphi_{t}$}

Here, we give the definition of the map $\varphi_{t}$ in Proposition 6.10. Fix $k=2, \ldots, l$. Suppose that $(\alpha ; \beta) \in \mathcal{H}(\lambda / \mu)$ has an even number $2 m$ of even $(k-1)$-overlaps $\left(\alpha_{i_{1}}, \beta_{i_{1}+k-1}\right), \ldots,\left(\alpha_{i_{2 m}}, \beta_{i_{2 m}+k-1}\right)\left(i_{1}<\cdots<i_{2 m}\right)$. We say $\left(\alpha_{i_{j}}, \beta_{i_{j}+k-1}\right)(1 \leq$ $j \leq 2 m)$ is of R-type if $j$ is odd, and of L-type if $j$ is even. Let $\left(\alpha_{i}, \beta_{i+k-1}\right)$ and $\left(\alpha_{i^{\prime}}, \beta_{i^{\prime}+k-1}\right)$ be a nearest pair of even $(k-1)$-overlaps with $i=i_{j}, i^{\prime}=i_{j+1}$. Then, we say the height 0 units $U \subset S_{+}$between $\beta_{i^{\prime}+k-1}$ and $\alpha_{i}^{*}$ and their duals $U^{*} \subset S_{-}$ are of $L R$-type if $j$ is odd, and of $R L$-type otherwise. Remark that any height $0 \mathrm{II}_{k}$-unit is either of LR-type or RL-type.

The next lemma is the key for the definition and the bijectivity of $\varphi_{t}$.

Lemma 6.16 $(\alpha ; \beta) \in \mathcal{H}(\lambda / \mu)$ does not have any odd $\mathrm{I}_{k-1}$-region if and only if the following conditions are satisfied:

(i) $(\alpha ; \beta)$ has an even number of even $(k-1)$-overlaps.

(ii) No connected component of $\tilde{\mathcal{U}}_{\mathrm{II}_{k}}$ of $(\alpha ; \beta)$ contains height $0 \mathrm{II}_{k}$-units of both LR- and RL-type, simultaneously.

(iii) Any connected component of $\tilde{\mathcal{U}}_{\mathrm{II}_{k}}$ of $(\alpha ; \beta)$ which contains a height $0 \mathrm{II}_{k}$-unit of LR-type is a $\mathrm{II}_{k}$-region.

The proofs of the lemma requires some graph-theoretical consideration, and it is given in Appendix 1.

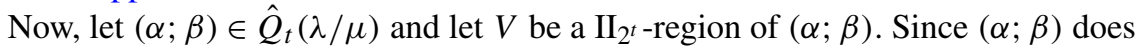
not have any odd $\mathrm{I}_{2^{t}-1}$-region by $(3)_{t}$, one of the following is satisfied by Lemma 6.16 with $k=2^{t}$ :

(a) All the height 0 units in $V$ are of LR-type.

(b) All the height 0 units in $V$ are of RL-type.

If (a) is satisfied, we say $V$ is of LR-type. See Fig. 11 for an example. We define the map $\varphi_{t}$ in Proposition 6.10 by (the composition of) the $2^{t}$-foldings with respect to all the $\mathrm{II}_{2^{t}}$-regions $V_{1}, \ldots, V_{p}$ of LR-type of $(\alpha ; \beta)$, i.e.,

$$
\varphi_{t}:(\alpha ; \beta) \mapsto \varepsilon_{V_{1}}^{2^{t}} \circ \cdots \circ \varepsilon_{V_{p}}^{2^{t}}(\alpha ; \beta)
$$

\subsection{Proof of Proposition 6.10}

To begin with, we give two lemmas.

Lemma 6.17 Let $(\alpha ; \beta) \in \hat{Q}_{t}(\lambda / \mu)$ and $W=V_{1} \cup \cdots \cup V_{p}$ for $V_{i}$ 's in (6.8). Then,

(1) $\alpha_{i}$ intersects with $W$ at height 0 if and only if

$$
\#\left\{j \mid j \leq i \text { and }\left(\alpha_{j}, \beta_{j+2^{t}-1}\right) \text { is an even }\left(2^{t}-1\right) \text {-overlap }\right\}
$$

is odd. 


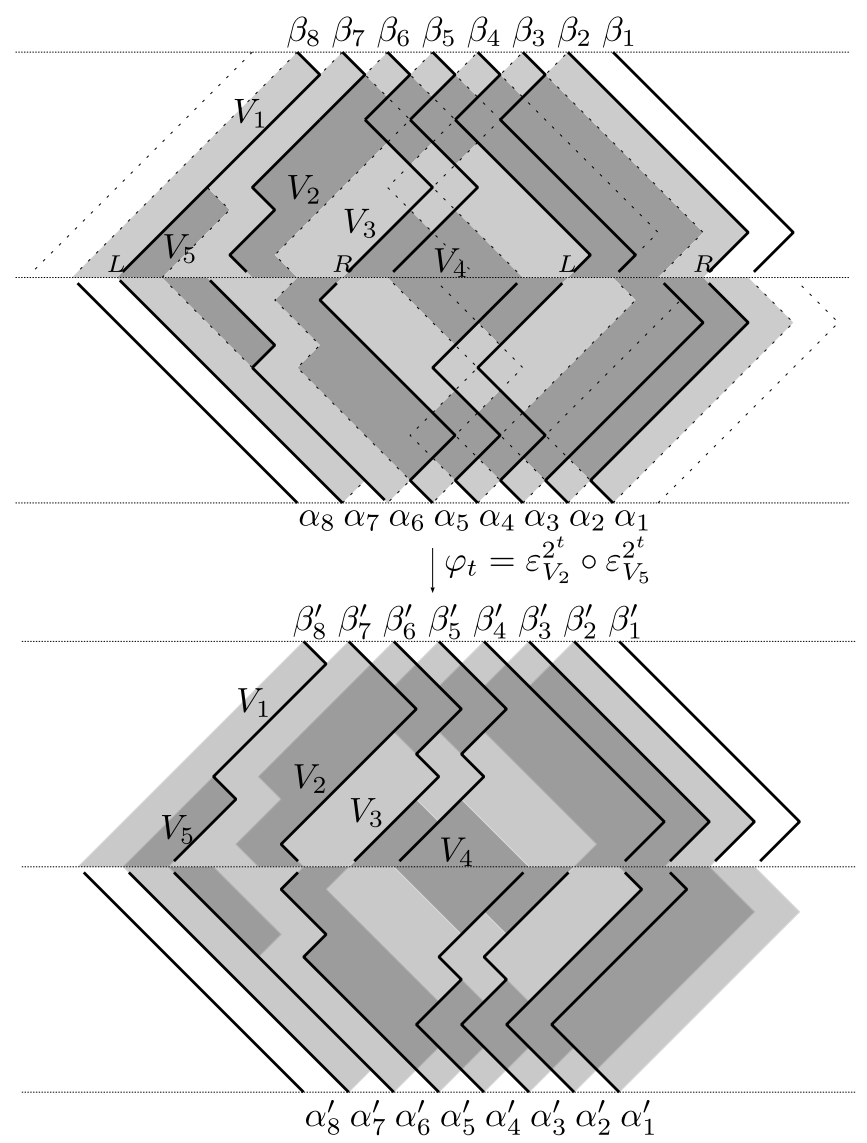

Fig. 11 An example of $(\alpha ; \beta) \in \hat{Q}_{t}(\lambda / \mu)$, its $\mathrm{I}_{2^{t}-1^{-}}$-regions $V_{1}, V_{3}$ and $\mathrm{II}_{2^{t}}$-regions $V_{2}, V_{4}, V_{5}$, and the map $\varphi_{t}$ for $t=1$. The even $\left(2^{t}-1\right)$-overlaps $\left(\alpha_{1}, \beta_{2}\right)$ and $\left(\alpha_{5}, \beta_{6}\right)$ are of R-type, $\left(\alpha_{3}, \beta_{4}\right)$ and $\left(\alpha_{7}, \beta_{8}\right)$ are of L-type, and $V_{2}$ and $V_{5}$ are $\mathrm{II}_{2} t$-regions of LR-type

(2) $\beta_{i}$ intersects with $W$ at height 0 if and only if

$$
\#\left\{j \mid j<i \text { and }\left(\alpha_{j-2^{t}+1}, \beta_{j}\right) \text { is an even }\left(2^{t}-1\right) \text {-overlap }\right\}
$$

is odd.

Proof We prove it for (1). Let $U \subset W$ be a height 0 unit which intersects with $\alpha_{i}$. Since $U$ is of LR-type, and the number of the even $\left(2^{t}-1\right)$-overlaps $\left(\alpha_{j}, \beta_{j+2^{t}-1}\right)$ to the right of $U$ is odd.

Conversely, suppose the above number is odd. Then, the unit $U$ whose right vertex intersects with $\alpha_{i}$ is of LR-type. Moreover, $U$ is a $\mathrm{II}_{2^{t}}$-unit because $\beta_{i+2^{t}}(0) \leq$ $\alpha_{i}(0)-2$, by Conditions (4) $t$ and $(6)_{t}$. Then, $U$ is included in $W$ by Lemma 6.16; therefore, $\alpha_{i}$ intersects with $W$.

Lemma 6.18 Let $V$ be a $\mathrm{II}_{k}$-region of $(\alpha ; \beta) \in \mathcal{H}(\lambda / \mu)$ and $\left(\alpha^{\prime} ; \beta^{\prime}\right)=\varepsilon_{V}^{k}(\alpha ; \beta)$. Then, 
(1) For any $r=1, \ldots, k$, both $\alpha_{i}$ and $\beta_{i+r}$ intersect with $V$ at height 0 and $\alpha_{i}(0) \geq$ $\beta_{i+r}(0)+2$ if and only if both $\alpha_{i+r-k}^{\prime}$ and $\beta_{i+k}^{\prime}$ intersect with $V$ at height 0 and $\alpha_{i+r-k}^{\prime}(0) \leq \beta_{i+k}^{\prime}(0)$.

(2) Suppose that $(\alpha ; \beta)$ does not have any odd $\mathrm{I}_{2 k-1}$-region. Then, $V^{\prime}$ is an odd $\mathrm{II}_{1}$-region of $(\alpha ; \beta)$ if and only if $V^{\prime}$ is an odd $\mathrm{II}_{1}$ - or odd $\mathrm{I}_{2 k-1}$-region of $\left(\alpha^{\prime} ; \beta^{\prime}\right)$.

Proof (1) It is obtained by (6.3). (2) If $V^{\prime}$ is an odd $\mathrm{II}_{1}$-region of $(\alpha ; \beta)$, then by Lemma 6.5 (2), $V^{\prime} \subset V$ or $V^{\prime} \cap V=\emptyset$. In the former case, $V^{\prime}$ is a $\mathrm{I}_{2 k-1}$-region of $\left(\alpha^{\prime} ; \beta^{\prime}\right)$ by Proposition $6.6(3)$. In the latter case, $V^{\prime}$ is also a $\mathrm{II}_{1}$-region of $\left(\alpha^{\prime} ; \beta^{\prime}\right)$. The converse is similar.

Now, let us prove $\varphi_{t}: \hat{Q}_{t}(\lambda / \mu) \rightarrow Q_{t+1}(\lambda / \mu)$ is a bijection. First, we prove $\varphi_{t}\left(\hat{Q}_{t}(\lambda / \mu)\right) \subset Q_{t+1}(\lambda / \mu)$. Set $\left(\alpha^{\prime} ; \beta^{\prime}\right)=\varphi_{t}(\alpha ; \beta)$. Let $V_{1}, \ldots, V_{p}$ be the set of all the $\mathrm{II}_{2^{t}}$-regions of LR-type of $(\alpha ; \beta)$, and set $W=V_{1} \cup \cdots \cup V_{p}$. We remark that $V_{i}$ is a $\mathrm{I}_{2^{t}}$-region of $\left(\alpha^{\prime} ; \beta^{\prime}\right)$ by Proposition 6.6 (3).

- (1) $)_{t+1}$. By the definition of the $k$-folding and Condition $(1)_{t}$ of $(\alpha ; \beta)$, we have $\alpha_{i}^{\prime}(0) \leq \alpha_{i}(0) \leq \beta_{i}(0) \leq \beta_{i}^{\prime}(0)$.

- $(2)_{t+1}$ and $(3)_{t+1}$. This is obtained by Conditions $(2)_{t}$ and $(4)_{t}$ of $(\alpha ; \beta)$ and Lemma 6.18 (2).

- (4) $)_{t+1}$. Suppose that $\left(\alpha_{i}^{\prime}, \beta_{i+2^{t+1}}^{\prime}\right)$ is a $2^{t+1}$-overlap of $\left(\alpha^{\prime} ; \beta^{\prime}\right)$. Since $\left(\alpha_{i}, \beta_{i+2^{t+1}}\right)$ is not a $2^{t+1}$-overlap by $(4)_{t}, \alpha_{i}^{\prime}$ and $\beta_{i+2^{t+1}}^{\prime}$ should intersect with $W$ at height 0 (otherwise, $\left.\left(\alpha_{i}, \beta_{i+2^{t+1}}\right)=\left(\alpha_{i}^{\prime}, \beta_{i+2^{t+1}}^{\prime}\right)\right)$. As (6.3), we have $\alpha_{i}^{\prime}(0)=\beta_{i+2^{t}}^{*}(0)$ and $\beta_{i+2^{t+1}}^{\prime}(0)=\alpha_{i+2^{t}}^{*}(0)$, and therefore, $\beta_{i+2^{t}}(0) \leq \alpha_{i+2^{t}}(0)-2$, which contradicts to Condition $(1)_{t}$ of $(\alpha ; \beta)$.

- $(5)_{t+1}$. Since $(\alpha ; \beta)$ satisfies $(7)_{t}$, there exists an even $\left(2^{t}-1\right)$-overlap $\left(\alpha_{i}, \beta_{i+2^{t}-1}\right)$. By Condition $(4)_{t},\left(\alpha_{i-1}, \beta_{i+2^{t}-1}\right)$ is not a $2^{t}$-overlap. If $\alpha_{i-1}(0)=$ $\beta_{i+2^{t}-1}(0)+1$, then $m_{t}\left(\alpha_{i-1}\right)=m_{t}\left(\beta_{i+2^{t}-1}\right)$, therefore, $\alpha_{i-1}(0) \equiv \beta_{i+2^{t}-1}(0)$ by Condition $(6)_{t}$, which is a contradiction. Thus, $\alpha_{i-1}(0) \geq \beta_{i+2^{t}-1}(0)+2$, and $\alpha_{i-1}$ and $\beta_{i+2^{t}-1}$ intersect with $W$ at height 0 . Therefore, by Lemma 6.18 (1), we have $\alpha_{i-1}^{\prime}(0) \leq \beta_{i+2^{t}-1}^{\prime}(0)$, namely, $\left(\alpha_{i-1}^{\prime}, \beta_{i+2^{t}-1}^{\prime}\right)$ is a $2^{t}$-overlap.

- (6) $)_{t+1}$. We prove $s\left(\alpha_{i}^{\prime}\right)=m_{t}\left(\alpha_{i}^{\prime}\right)$ (the proof for $s\left(\beta_{i}^{\prime}\right)=m_{t}\left(\beta_{i}^{\prime}\right)$ is similar).

Case 1. If $\alpha_{i}$ does not intersect with $W$ at height 0 , which implies $\alpha_{i}^{\prime}(0)=\alpha_{i}(0)$, then $s\left(\alpha_{i}^{\prime}\right)=s\left(\alpha_{i}\right)$. On the other hand, we have

$$
m_{t+1}\left(\alpha_{i}^{\prime}\right)-m_{t}\left(\alpha_{i}\right)=-\#\left\{j \mid j \leq i,\left(\alpha_{j}, \beta_{j+2^{t}-1}\right) \text { is an even }\left(2^{t}-1\right) \text {-overlap }\right\},
$$

by Lemma 6.18 (1) and Condition $(4)_{t}$ of $(\alpha ; \beta)$. Moreover, the right-hand side of (6.9) is even by Lemma 6.17 (1). By Condition $(6)_{t}$ of $(\alpha ; \beta)$, we obtain $m_{t+1}\left(\alpha_{i}^{\prime}\right) \equiv$ $m_{t}\left(\alpha_{i}\right) \equiv s\left(\alpha_{i}\right)=s\left(\alpha_{i}^{\prime}\right)$.

Case 2. If $\alpha_{i}$ intersects with $W$ at height 0 , which implies that $\alpha_{i}^{\prime}(0)=\beta_{i+2^{t}}^{*}(0)$, then $s\left(\alpha_{i}^{\prime}\right)=s\left(\beta_{i+2^{t}}\right)+1$. On the other hand, we have

$$
m_{t+1}\left(\alpha_{i}^{\prime}\right)-m_{t}\left(\beta_{i+2^{t}}\right)=-\#\left\{j \mid j<i,\left(\alpha_{j-2^{t}+1}, \beta_{j}\right) \text { is an even }\left(2^{t}-1\right) \text {-overlap }\right\},
$$

by Lemma 6.18 (1) and Condition $(4)_{t}$ of $(\alpha ; \beta)$. Moreover, the right-hand side of (6.9) is odd by Lemma 6.17 (1). By Condition $(6)_{t}$ of $(\alpha ; \beta)$, we obtain $m_{t+1}\left(\alpha_{i}^{\prime}\right) \not \equiv$ $m_{t}\left(\beta_{i+2^{t}}\right) \equiv s\left(\beta_{i+2^{t}}\right) \not \equiv s\left(\alpha_{i}^{\prime}\right)$. 
Next, let us define the inverse map

$$
\varphi_{t}^{-1}: Q_{t+1}(\lambda / \mu) \rightarrow \hat{Q}_{t}(\lambda / \mu)
$$

as follows: For any $\left(\alpha^{\prime} ; \beta^{\prime}\right) \in Q_{t+1}(\lambda / \mu)$, set

$$
\varphi_{t}^{-1}:\left(\alpha^{\prime} ; \beta^{\prime}\right) \mapsto(\alpha ; \beta):=\varepsilon_{V_{1}}^{2^{t}} \circ \cdots \circ \varepsilon_{V_{p}}^{2^{t}}\left(\alpha^{\prime} ; \beta^{\prime}\right),
$$

where $V_{1}, \ldots, V_{p}$ are the set of all the $\mathrm{I}_{2^{t}}$-regions of $\left(\alpha^{\prime} ; \beta^{\prime}\right)$. By $(5)_{t+1}$, we have $p \geq 1$. Set $W=V_{1} \cup \cdots \cup V_{p}$. We prove that $(\alpha ; \beta) \in \hat{Q}_{t}(\lambda / \mu)$.

- (1) $t_{t}$. By (6.3) wherein $\left(\alpha_{i}\right),\left(\alpha_{i}^{\prime}\right)$ and $\left(\beta_{i}\right),\left(\beta_{i}^{\prime}\right)$ are interchanged, $\alpha_{i}(0)=$ $\beta_{i+2^{t}}^{\prime *}(0)$ or $\alpha_{i}^{\prime}(0)$, and $\beta_{i}(0)=\alpha_{i-2^{t}}^{\prime *}(0)$ or $\beta_{i}^{\prime}(0)$. Only the case $\left(\alpha_{i}(0), \beta_{i}(0)\right)=$ $\left(\beta_{i+2^{t}}^{\prime *}(0), \alpha_{i-2^{t}}^{\prime *}(0)\right)$ is nontrivial. By $(4)_{t+1}$, we have $\alpha_{i-2^{t}}^{\prime}(0)>\beta_{i+2^{t}}^{\prime}(0)$. Then, we have $\alpha_{i-2^{t}}^{\prime}(0) \leq \beta_{i+2^{t}}^{\prime}(0)$ by the same argument in the proof of $(5)_{t+1}$ of $\varphi_{t}(\alpha ; \beta)$.

- (2) $)_{t}$. This is because of Lemma 6.18 (2) and the Conditions (2) $)_{t+1}$ and (3) $t+1$ of $\left(\alpha^{\prime} ; \beta^{\prime}\right)$.

- (4) $)_{t}$. Suppose that $(\alpha ; \beta)$ has a $2^{t}$-overlap $\left(\alpha_{i}, \beta_{i+2^{t}}\right)$. By Proposition 6.6 (2), $V_{1}, \ldots, V_{p}$ are $\mathrm{II}_{2^{t}}$-regions of $(\alpha ; \beta)$, and therefore, $\alpha_{i}$ and $\beta_{i+2^{t}}$ do not intersect with $W$ at height 0 by Lemma 6.2 (3). Then, $\left(\alpha_{i}^{\prime}, \beta_{i+2^{t}}^{\prime}\right)$ is also a $2^{t}$-overlap because $\alpha_{i}^{\prime}(0)=\alpha_{i}(0)$ and $\beta_{i+2^{t}}^{\prime}(0)=\beta_{i+2^{t}}(0)$, which implies that there exists a height 0 $\mathrm{I}_{2^{t}}$-unit $U \not \subset W$ of $\left(\alpha^{\prime} ; \beta\right)$ between $\alpha_{i}^{\prime}$ and $\beta_{i+2^{t}}^{\prime}$, and then contradicts to the definition of $W$.

- (3) $t$ and $(7)_{t}$. This is the most non-trivial part of the proof of Proposition 6.10. We prove that Conditions (i)-(iii) in Lemma 6.16 (for $k=2^{t}$ ) are satisfied.

Let us study when a pair $\left(\alpha_{i}, \beta_{i+2^{t}-1}\right)$ is an even $\left(2^{t}-1\right)$-overlap. There are four cases $\mathrm{A}-\mathrm{D}$ to be considered. We prove that $\left(\alpha_{i}, \beta_{i+2^{t}-1}\right)$ is even only in Cases $\mathrm{A}$ and $\mathrm{B}$.

A. The case where $\alpha_{i}$ does not intersect with $W$ at height 0 and $\beta_{i+2^{t}-1}$ intersects with $W$ at height 0 . In this case, we have $\beta_{i+2^{t}-1}^{*}(0)=\alpha_{i-1}^{\prime}(0)$ and $\alpha_{i}(0)=$ $\alpha_{i}^{\prime}(0)$, and then $\left(\alpha_{i}, \beta_{i+2^{t}-1}\right)$ is a $\left(2^{t}-1\right)$-overlap. We also have $m_{t+1}\left(\alpha_{i}^{\prime}\right)=$ $m_{t+1}\left(\alpha_{i-1}^{\prime}\right)$. This is because $\left(\alpha_{i-1}^{\prime}, \beta_{i}^{\prime}\right)$ is not an even 1-hole, and $\left(\alpha_{i}^{\prime}, \beta_{i+2^{t+1}-1}^{\prime}\right)$ is not an (even) $\left(2^{t+1}-1\right)$-overlap (otherwise, $\left(\alpha_{i}^{\prime}, \beta_{i+2^{t}}^{t}\right)$ is also a $2^{t}$-overlap, and $\alpha_{i}$ intersects with $W$ at height 0 , which contradicts to the assumption). Therefore, $s\left(\alpha_{i}^{\prime}\right) \equiv s\left(\alpha_{i-1}^{\prime}\right)$, i.e., $\alpha_{i}^{\prime}(0) \not \equiv \alpha_{i-1}^{\prime}(0)$. So, $\left(\alpha_{i}, \beta_{i+2^{t}-1}\right)$ is an even $\left(2^{t}-1\right)$ overlap.

B. The case where $\alpha_{i}$ intersects with $W$ at height 0 and $\beta_{i+2^{t}-1}$ does not intersect with $W$ at height 0 . As in Case $\mathrm{A}$, one can show that $\left(\alpha_{i}, \beta_{i+2^{t}-1}\right)$ is an even $\left(2^{t}-1\right)$-overlap.

C. The case where both $\alpha_{i}$ and $\beta_{i+2^{t}-1}$ intersect with $W$ at height 0 . In this case, we have $\beta_{i+2^{t}-1}^{*}(0)=\alpha_{i-1}^{\prime}(0), \alpha_{i}^{*}(0)=\beta_{i+2^{t}}^{\prime}(0)$, and $\left(\alpha_{i-1}^{\prime}, \beta_{i+2^{t}-1}^{\prime}\right),\left(\alpha_{i}^{\prime}, \beta_{i+2^{t}}^{\prime}\right)$ are $2^{t}$-overlaps. If $\left(\alpha_{i}, \beta_{i+2^{t}-1}\right)$ is a $\left(2^{t}-1\right)$-overlap, then there does not exist any path at height 0 between $\beta_{i+2^{t}}^{\prime}(0)$ and $\alpha_{i-1}^{\prime}(0)$. We have $m_{t+1}\left(\alpha_{i-1}^{\prime}\right)=$ $m_{t+1}\left(\beta_{i+2^{t}}^{\prime}\right)$, therefore, $s\left(\alpha_{i-1}^{\prime}\right) \equiv s\left(\beta_{i+2^{t}}^{\prime}\right)$, namely, $\left(\alpha_{i}, \beta_{i+2^{t}-1}\right)$ is odd.

D. The case where both $\alpha_{i}$ and $\beta_{i+2^{t}-1}$ do not intersect with $W$ at height 0 . In this case, we have $\alpha_{i}(0)=\alpha_{i}^{\prime}(0)$ and $\beta_{i+2^{t}-1}^{\prime}(0)=\beta_{i+2^{t}-1}(0)$. If $\left(\alpha_{i}, \beta_{i+2^{t}-1}\right)$ is a $\left(2^{t}-1\right)$-overlap, then there does not exist any path at height 0 between $\alpha_{i}^{\prime}$ and 
$\beta_{i+2^{t}-1}^{\prime}$ because of the assumption. Then we have $m_{t+1}\left(\beta_{i+2^{t}-1}^{\prime}\right) \equiv m_{t+1}\left(\alpha_{i}^{\prime}\right)$, therefore, $\left(\alpha_{i}, \beta_{i+2^{t}-1}\right)$ is odd.

To summarize, the even $\left(2^{t}-1\right)$-overlaps of L-type (resp. R-type) of $(\alpha ; \beta)$ are the ones in Case A (resp. Case B); furthermore, the number of the even $\left(2^{t}-1\right)$-overlaps of $(\alpha ; \beta)$ is even, and a height $0 \mathrm{II}_{2^{t}}$-unit $U$ of $(\alpha ; \beta)$ is of LR-type if and only if $U \subset W$. Also, $W$ is a union of $\mathrm{II}_{2^{t}}$-regions of $(\alpha ; \beta)$ by Proposition 6.6 (2). Thus, Conditions (i)-(iii) in Lemma 6.16 (for $k=2^{t}$ ) are satisfied.

- (5) $t$. If $\left(\alpha_{i}, \beta_{i+2^{t}-1}\right)$ is an overlap, then $\left(\alpha_{i}, \beta_{i+2^{t-1}}\right)$ is also an overlap, and therefore, $(5)_{t}$ holds.

- (6) $t$. We prove $s\left(\alpha_{i}\right) \equiv m_{t}\left(\alpha_{i}\right)$.

1. The case where $\alpha_{i}$ does not intersect with $W$ at height 0 . This is the cases $\mathrm{A}$ and $\mathrm{D}$ in the proof of (3) $)_{t}$. By $\alpha_{i}(0)=\alpha_{i}^{\prime}(0)$, we have $s\left(\alpha_{i}\right)=s\left(\alpha_{i}^{\prime}\right)$. Since every even $\left(2^{t+1}-1\right)$-overlap of $\left(\alpha^{\prime} ; \beta^{\prime}\right)$ intersects with $W$ at height 0 , we have $\left(\alpha_{i}, \beta_{i+1}\right)$ is an even 1 -hole by Lemma 6.18 (1). Therefore,

$$
m_{t}\left(\alpha_{i}\right)-m_{t+1}\left(\alpha_{i}^{\prime}\right)=\#\left\{j \mid j \leq i,\left(\alpha_{j}, \beta_{j+2^{t}-1}\right) \text { is an even }\left(2^{t}-1\right) \text {-overlap }\right\} .
$$

From the proof of (3) $)_{t}$, the right-hand side of (6.11) is even. Thus, $m_{t}\left(\alpha_{i}\right) \equiv s\left(\alpha_{i}\right)$.

2. The case where $\alpha_{i}$ intersects with $W$ at height 0 . This is the cases $\mathrm{B}$ and $\mathrm{C}$ in the proof of $(3)_{t}$. By $\alpha_{i}^{*}(0)=\beta_{i+2^{t}}^{\prime}(0)$ and $\beta_{1}^{\prime}(0)=\beta_{1}(0)$, we have $s\left(\alpha_{i}\right) \not \equiv s\left(\beta_{i+2^{t}}^{\prime}\right)$. As in the former case, we have

$$
m_{t}\left(\alpha_{i}\right)-m_{t+1}\left(\beta_{i+2^{t}}^{\prime}\right)=\#\left\{j \mid j \leq i,\left(\alpha_{j}, \beta_{j+2^{t}-1}\right) \text { is an even }\left(2^{t}-1\right) \text {-overlap }\right\},
$$

and the right-hand side of (6.12) is odd. Thus, $m_{t}\left(\alpha_{i}\right) \not \equiv m_{t+1}\left(\beta_{i+2^{t}}^{\prime}\right)$, which implies $m_{t}\left(\alpha_{i}\right) \equiv s\left(\alpha_{i}\right)$.

Finally, the fact that $\pi^{-1}$ is the inverse of $\pi$ is obvious by construction.

\subsection{Proof of Proposition 6.12}

Finally, we show that $\phi$ is weight-preserving and then complete the proof of Proposition 5.1.

For $p \in P_{2}(\lambda / \mu)$ and $p^{\prime}=\phi(p) \in P(\lambda / \mu)$, we shall show that $z_{a}^{p}=z_{a}^{p^{\prime}}$.

Let $(\alpha ; \beta) \in Q_{1}(\lambda / \mu)$ and $\left(\alpha^{\prime} ; \beta^{\prime}\right) \in R(\lambda / \mu)$ be the corresponding ones to $p$ and $p^{\prime}$ under the identification $\pi$. We decompose the monomial $z_{a}^{p}$ in (3.4) into two parts as $z_{a}^{p}=H E$, where $H$ is the factor coming from the lower and upper paths $(\alpha ; \beta)$ for $p$, and $E$ is the one from the height 0 parts (E-steps) of $p$. We do the same for $z_{a}^{p^{\prime}}$ as $z_{a}^{p^{\prime}}=H^{\prime} E^{\prime}$.

By a similar argument for the proof of Proposition 4.12, one obtains that

$$
H^{\prime}=H \delta^{-1},
$$

where

$$
\delta=\prod_{i=1}^{l}\left(\prod_{k=\alpha_{i}^{\prime}(0)}^{\alpha_{i}(0)-1} z_{n, a-2 k} \prod_{k=\beta_{i}(0)}^{\beta_{i}^{\prime}(0)-1} z_{\bar{n}, a-2 k}\right)=\prod_{i=1}^{l} \prod_{k=\beta_{i}(0)}^{\beta_{i}^{\prime}(0)-1} z_{\bar{n}, a-2 k} z_{n, a-2 k-2} .
$$


Therefore, it is enough to show that

$$
E^{\prime}=E \delta
$$

To see it, we notice two facts following from (6.14), and the definitions of $E, E^{\prime}$, and $\phi$ :

Fact 1. Both $E^{\prime}$ and $E \delta$ are products of the factors $z_{\bar{n}, a-2 k} z_{n, a-2 k+2}$ with $k \in \mathbb{Z}$.

Fact 2. For each $k \in \mathbb{Z}$, the total degree of $z_{\bar{n}, a-2 k}$ and $z_{n, a-2 k}$ for $E^{\prime}$ and the one for $E \delta$ are equal.

Now, the equality (6.15) easily follows from these facts.

\section{Appendix 1 Proof of Lemma 6.16}

In this section, we prove Lemma 6.16. To do so, we use a lemma (Lemma 7.3) for a certain graph.

Let $H \subset \mathbb{R}^{2}$ be the upper half plane. A graph $\Gamma$ consists of a set $V(\Gamma)$ of vertices on the boundary of $H$, denoted by $\times$, and a set $A(\Gamma)$ of $\operatorname{arcs}$ in $H$ which satisfies the following conditions:

(i) An arc in $A(\Gamma)$ connects two vertices in $V(\Gamma)$.

(ii) In each side of a vertex $v$, the number of the vertices which are connected with $v$ is at most one.

(iii) The arcs are nonintersecting with each other at $H \backslash V(\Gamma)$.

The vertices are labeled as $L, R, L, R, \ldots$ from left to right. The following is an example of a graph:

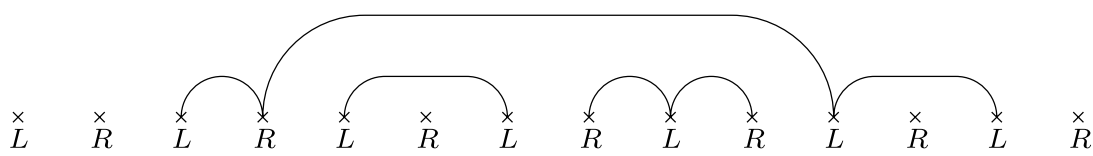

Let $v, v^{\prime} \in V(\Gamma)$. If $v$ and $v^{\prime}$ are connected by an arc in $A(\Gamma)$, then we write $v \frown v^{\prime}$. Then $\frown$ generates an equivalence relation $\sim$ in $V(\Gamma)$. We call each equivalence class $S$ of $V(\Gamma)$ a segment of $\Gamma$. We say that a segment $S$ is even (resp. odd) if the number of vertices $|S|$ is even (resp. odd).

Let $S$ and $S^{\prime}$ be segments of $\Gamma$, and let $v_{1}$ and $v_{2}$ be the leftmost and the rightmost vertices in $S$. If all the vertices $v \in S$ are between $v_{1}$ and $v_{2}$, then we say that $S^{\prime}$ is inside $S$. Let us write $S^{\prime} \triangleleft S$ if $S^{\prime}$ is inside $S$.

Lemma 7.1 A graph $\Gamma$ does not have any odd segment if and only if each segment $S$ of $\Gamma$ satisfies one of the following conditions:

(1) The leftmost vertex is $L$ and the rightmost vertex is $R$.

(2) The leftmost vertex is $R$ and the rightmost vertex is $L$.

Proof $(\Rightarrow)$ Let $S$ be an even segment of $\Gamma$ and let $v_{1}$ and $v_{2}$ be its leftmost and the rightmost vertices. We can show, by induction with respect to $\triangleleft$, that the number 
$h(S)$ of the vertices between $v_{1}$ and $v_{2}$ is even, which implies that $S$ satisfies (1) or (2).

$(\Leftarrow)$ If $\Gamma$ has an odd segment, then there exists an odd segment $S$ which does not have any odd segment inside. Since the number $h(S)$ is odd, $S$ satisfies neither (1) nor (2).

For any graph $\Gamma$, we define the dual graph $\Gamma^{*}=\left(V\left(\Gamma^{*}\right), A\left(\Gamma^{*}\right)\right)$ as follows: Let $V\left(\Gamma^{*}\right)$ be the set of all the vertices $\diamond$ which are placed between each nearest pair of vertices in $V(\Gamma)$, and the vertex ' $\infty$ ' which is on the right to the rightmost vertex of $\Gamma$. Let $A\left(\Gamma^{*}\right)$ be the set of all the dotted arcs which connects each nearest pair of vertices in $V\left(\Gamma^{*}\right)$ without intersecting with the arcs in $A\left(\Gamma^{*}\right)$. For example, the dual graph $\Gamma^{*}$ of $\Gamma$ in (7.1) is given as follows:

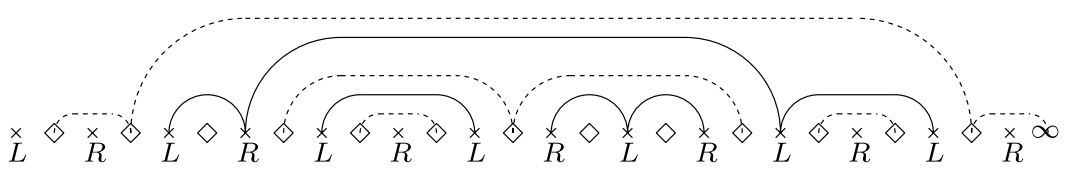

A segment $S^{*}$ of $\Gamma^{*}$ is similarly defined as that of $\Gamma$. We say the vertices of $\Gamma^{*}$ between $L$ and $R$ (resp. $R$ and $L$ ) is of LR-type (resp. of $R L$-type).

Definition 7.2 Let $S^{*}$ be a segment of $\Gamma^{*}$. Then,

(1) We say that $S^{*}$ is mixed if it contains vertices of both LR-type and RL-type simultaneously, and unmixed otherwise. We say an unmixed segment $S^{*}$ is LR-type (resp. RL-type) if it consists of vertices of LR-type (resp. RL-type) (also, $\infty$ may be included).

(2) We say that $S^{*}$ is bounded if it does not contain $\infty$.

Lemma 7.3 A graph $\Gamma$ does not have any odd segment if and only if the following conditions are satisfied:

(i) $|V(\Gamma)|$ is even.

(ii) $\Gamma^{*}$ is unmixed.

(iii) Any segment of LR-type of $\Gamma^{*}$ is bounded.

Proof $(\Rightarrow)$ (ii) If there exists a mixed segment of $\Gamma^{*}$, then one of the following occurs, which contradicts to Lemma 7.1:
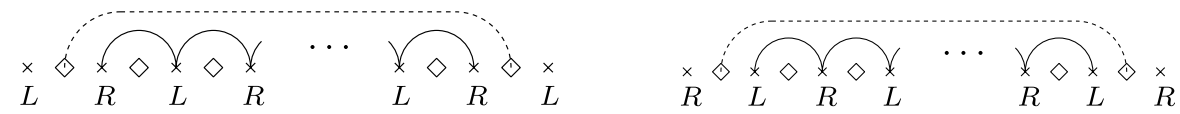

(iii) If there exits an unmixed segment of LR-type which is not bounded, then there exists an LR-type vertex $w \in V\left(\Gamma^{*}\right)$ which belongs to the same segment with $\infty$.

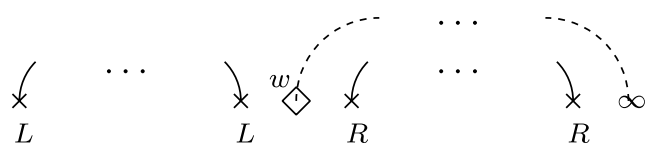


The number of all the vertices to the left of $w$ is odd, which means that there exists an odd segment of $\Gamma$ to the left of $w$.

$(\Leftarrow)$ If there exists an odd segment in $\Gamma$, then there exists a segment $S$ of $\Gamma$ that satisfies one of the following conditions, by Lemma 7.1:

(1) Both the leftmost and the rightmost vertices of $S$ are $L$.

(2) Both the leftmost and the rightmost vertices of $S$ are $R$.

For example, suppose that $S$ satisfies (1), and let $v \in V\left(\Gamma^{*}\right)$ be the one right-next to $S$. Then, one of the following occurs (by (i)):

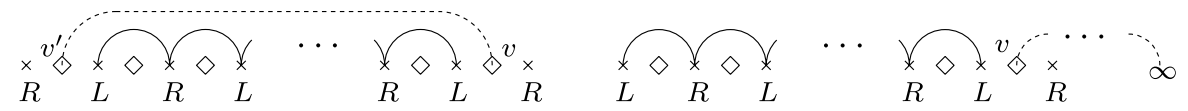

Namely, if there exists an RL-type vertex $v^{\prime} \in V\left(\Gamma^{*}\right)$ left-next to $S$, then $v$ and $v^{\prime}$ belongs to the same segment of $\Gamma^{*}$, otherwise, $v$ belongs to the same segment with the vertex $\infty$. The (2) case is similar.

Now, let us prove Lemma 6.16. With any $(\alpha ; \beta) \in \mathcal{H}(\lambda / \mu)$, we associate a graph $\Gamma$ as follows: Each vertex of $\Gamma$ naturally corresponds to each even $(k-1)$-overlaps of $(\alpha ; \beta)$. An arc of $\Gamma$ connects a nearest pair of even $(k-1)$-overlaps (under the above correspondence) belonging to the same $\mathrm{I}_{k-1}$-region. Then, an odd segment of $\Gamma$ corresponds to an odd $\mathrm{I}_{k-1}$-region of $(\alpha ; \beta)$. Furthermore, Conditions (i), (ii), and (iii) of Lemma 7.3 are equivalent to the ones of Lemma 6.16 due to the complementarity of the $I_{k-1}$ - and the $\mathrm{II}_{k}$-units (Lemma 6.2 (4)). This completes the proof of Lemma 6.16.

\section{References}

1. Bazhanov, V.V., Reshetikhin, N.: Restricted solid-on-solid models connected with simply laced algebras and conformal field theory. J. Phys. A 23, 1477-1492 (1990)

2. Chari, V., Moura, A.: Characters of fundamental representations of quantum affine algebras. Acta Appl. Math. 90, 43-63 (2006)

3. Chari, V., Pressley, A.: Quantum affine algebras. Commun. Math. Phys. 142, 261-283 (1991)

4. Drinfel'd, V.: Hopf algebras and the quantum Yang-Baxter equation. Soviet. Math. Dokl. 32, 254-258 (1985)

5. Drinfel'd, V.: A new realization of Yangians and quantized affine algebras. Soviet. Math. Dokl. 36, 212-216 (1988)

6. Frenkel, E., Mukhin, E.: Combinatorics of $q$-characters of finite-dimensional representations of quantum affine algebras. Commun. Math. Phys. 216, 23-57 (2001)

7. Frenkel, E., Reshetikhin, N.: The $q$-characters of representations of quantum affine algebras and deformations of $\mathcal{W}$-algebras. Contemp. Math. 248, 163-205 (1999)

8. Fulmek, M., Krattenthaler, C.: Lattice path proofs for determinantal formulas for symplectic and orthogonal characters. J. Combin. Theory Ser. A 77, 3-50 (1997)

9. Gessel, I., Viennot, G.: Binomial determinants, paths, and hook length formulae. Adv. Math. 58, 300321 (1985)

10. Hernandez, D.: The Kirillov-Reshetikhin conjecture and solutions of T-systems. J. Reine Angew. Math. 596, 63-87 (2006)

11. Jimbo, M.: A $q$-difference analogue of $U(\hat{\mathfrak{g}})$ and the Yang-Baxter equation. Lett. Math. Phys. 10, 63-69 (1985)

12. Kashiwara, M., Nakashima, T.: Crystal graphs for representations of the $q$-analogue of classical Lie algebras. J. Algebra 165, 295-345 (1994) 
13. Koike, K., Terada, I.: Young-diagrammatic methods for the representation theory of the classical groups of type $B_{n}, C_{n}, D_{n}$.J. Algebra 107, 466-511 (1987)

14. Kuniba, A., Ohta, Y., Suzuki, J.: Quantum Jacobi-Trudi and Giambelli formulae for $U_{q}\left(B_{r}^{(1)}\right)$ from the analytic Bethe ansatz. J. Phys. A 28, 6211-6226 (1995)

15. Kuniba, A., Suzuki, J.: Analytic Bethe ansatz for fundamental representations of Yangians. Commun. Math. Phys. 173, 225-264 (1995)

16. Macdonald, I.G.: Symmetric Functions and Hall Polynomials, 2nd edn. Oxford University Press, New York (1995)

17. Nakai, W., Nakanishi, T.: Paths, tableaux and $q$-characters of quantum affine algebras: the $C_{n}$ case. J. Phys. A: Math. Gen. 39, 2083-2115 (2006)

18. Nakai, W., Nakanishi, T.: Paths and tableaux descriptions of Jacobi-Trudi determinant associated with quantum affine algebra of type $C_{n}$. Preprint, math.QA/0604158

19. Nakajima, H.: $t$-analogs of $q$-characters of quantum affine algebras of type $A_{n}$ and $D_{n}$. Contemp. Math. 325, 141-160 (2003)

20. Okado, M., Schilling, A., Shimozono, M.: Virtual crystals and fermionic formulas of type $D_{n+1}^{(2)}$, $A_{2 n}^{(2)}$, and $C_{n}^{(1)}$. Represent. Theory 7, 101-163 (2003)

21. Schilling, A., Sternberg, P.: Finite-dimensional crystals $B^{2, s}$ for quantum affine algebras of type $D_{n}^{(1)}$. J. Algebr. Comb. 23, 317-354 (2006) 